\title{
The grazing collision limit of the inelastic Kac model around a Lévy-type equilibrium.
}

\author{
G. Furioli ${ }^{*}$ A. Pulvirenti $\nmid$ E. Terraneo $\ddagger$ and G. Toscani ${ }^{\S}$
}

November 12, 2018

\begin{abstract}
This paper is devoted to the grazing collision limit of the inelastic Kac model introduced in PT04, when the equilibrium distribution function is a heavy-tailed Lévy-type distribution with infinite variance. We prove that solutions in an appropriate domain of attraction of the equilibrium distribution converge to solutions of a Fokker-Planck equation with a fractional diffusion operator.
\end{abstract}

Keywords. Dissipative kinetic models, Lévy-type distributions, Fractional diffusion equations.

\section{Introduction}

The inelastic Kac model has been introduced in PT04, with the aim of obtaining a physically consistent one-dimensional dissipative kinetic model, sufficiently rich to exhibit a variety of steady states and similarity solutions. This model can be viewed as a dissipative generalization of the Kac caricature of a Maxwell gas introduced in the fifties [Kac59]. Kac model has been fruitfully used from that time on, to find explicit rates of convergence towards the Maxwellian equilibrium [McK66, TV99, since its simple structure (with respect to the full Boltzmann equation) makes possible to carry out exact computations. The inelastic Kac model reads

$$
\partial_{t} f(v, t)=Q_{p}(f, f)(v, t), \quad v \in \mathbb{R}, t \geq 0
$$

where the right-hand side of (11) describes the rate of change of the density function $f$ due to dissipative collisions,

$$
Q_{p}(f, f)(v, t)=\int_{\mathbb{R} \times[-\pi / 2, \pi / 2]} b(\theta)\left[\chi^{-1} f\left(v_{p}^{* *}, t\right) f\left(w_{p}^{* *}, t\right)-f(v, t) f(w, t)\right] \mathrm{d} \theta \mathrm{d} w .
$$

\footnotetext{
*University of Bergamo, viale Marconi 5, 24044 Dalmine, Italy. giulia.furioli@unibg.it

${ }^{\dagger}$ Department of Mathematics, University of Pavia, via Ferrata 1, 27100 Pavia, Italy. ada.pulvirenti@unipv.it

${ }^{\ddagger}$ Department of Mathematics, University of Milano, via Saldini 50, 20133 Milano, Italy. Elide.Terraneo@mat.unimi.it

${ }^{\S}$ Department of Mathematics, University of Pavia, via Ferrata 1, 27100 Pavia, Italy. giuseppe.toscani@unipv.it
} 
The kernel $b$ is an integrable function taking values in $[-\pi / 2, \pi / 2]$, which describes the details of the possible outcomes in the binary collision. The velocities $\left(v_{p}^{* *}, w_{p}^{* *}\right)$ are the pre collision velocities of the so-called inverse collision, which results with $(v, w)$ as post collision velocities. Given $(v, w)$, the post collision velocities $\left(v_{p}^{*}, w_{p}^{*}\right)$ are defined simply generalizing the Kac rule

$$
v_{p}^{*}=v \cos \theta|\cos \theta|^{p}-w \sin \theta|\sin \theta|^{p}, \quad w_{p}^{*}=v \sin \theta|\sin \theta|^{p}+w \cos \theta|\cos \theta|^{p} .
$$

In (3) the positive constant $p<+\infty$ measures the degree of inelasticity. If $p=0$, the binary collision is elastic and we obtain the classical Kac equation, where the post collisional velocities are given by a rotation in the $(v, w)$ plane. The factor $\chi=|\sin \theta|^{2+2 p}+|\cos \theta|^{2+2 p}$ in (2) appears from the Jacobian of the transformation $\mathrm{d} v_{p}^{* *} \mathrm{~d} w_{p}^{* *}$ into $\mathrm{d} v \mathrm{~d} w$. The loss of energy in a single binary collision depends on the choice of the inelasticity parameter $p$, and it is given by

$$
\left(v_{p}^{*}\right)^{2}+\left(w_{p}^{*}\right)^{2}=\left(v^{2}+w^{2}\right)\left(|\sin \theta|^{2+2 p}+|\cos \theta|^{2+2 p}\right) .
$$

The structure of the inelastic Kac equation is similar to the inelastic Boltzmann equation for a Maxwell gas, and also here mass is conserved, while energy is non-increasing.

Resorting to Bobylev's argument [Bob88, the dissipative Kac equation can be fruitfully written in Fourier variables as

$$
\partial_{t} \widehat{f}(\xi, t)=\widehat{Q}_{p}(\widehat{f}, \widehat{f})(\xi, t),
$$

where $\widehat{f}(\xi, t)$ is the Fourier transform of $f(v, t)$ with respect to $v$

$$
\widehat{f}(\xi, t)=\int_{\mathbb{R}} e^{-i \xi v} f(v, t) \mathrm{d} v,
$$

and

$$
\widehat{Q}_{p}(\widehat{f}, \widehat{f})(\xi, t)=\int_{-\frac{\pi}{2}}^{\frac{\pi}{2}} b(\theta)\left[\widehat{f}\left(\xi_{p}^{+}, t\right) \widehat{f}\left(\xi_{p}^{-}, t\right)-\widehat{f}(\xi, t) \widehat{f}(0, t)\right] \mathrm{d} \theta
$$

In (6)

$$
\xi_{p}^{+}=\xi \cos \theta|\cos \theta|^{p}, \quad \xi_{p}^{-}=\xi \sin \theta|\sin \theta|^{p} .
$$

The Fourier description allows to verify that the inelastic Kac model, unlike what happens for the elastic model, possesses Maxwellian equilibria with infinite energy. Indeed, the collision operator (6) vanishes by choosing

$$
\widehat{M}_{p}(\xi)=\exp \left\{-\alpha|\xi|^{2 /(1+p)}\right\}, \quad \alpha>0 .
$$

In case $0<p \leq 1$, the function (8) represents a Lévy symmetric stable distribution of order $2 /(1+p)$ [Fel71. Consequently, the problem of convergence of the solution to (1D) to these equilibria for large times is deeply connected with the central limit theorem for stable laws, like the classical central limit theorem is closely connected with the convergence towards equilibrium of the elastic Boltzmann or Kac equation [LR79]. The 
main result in PT04 is that these Maxwellian equilibria (8) only attract solutions corresponding to initial data which are in a suitable (small) domain of attraction of (8). In particular, the energy of these initial data has to be unbounded.

As a matter of fact, the classical problem of the cooling of the dissipative gas is concerned with initial densities of finite energy [Vil06]. In this relevant physical case, the stationary solution is a Dirac delta function concentrated in $v=0$, which is nothing but a particular case of equilibrium (8) corresponding to $\alpha=0$. This equilibrium is strongly attractive, and its domain of attraction is given by all initial densities with bounded energy [Vil06].

If on the contrary the energy of the initial datum is unbounded, the collision mechanism given by (3) is in general not enough to cool down the gas towards a density with zero energy. In this second case however, convergence to Maxwellian equilibria of type (8) is possible. Hence, the existence of these equilibria is strictly linked to the weakness of the particular one-dimensional collision mechanism of the dissipative Kac equation.

The grazing limit procedure consists in letting the kernel $b(\theta)$ to concentrate on $\theta=0$ (which implies $v_{p}^{*}=v, w_{p}^{*}=w$ ) in such a way that the contribution of the collision integral does not vanish. This can be done by assuming on a family $\left\{b_{\varepsilon}\right\}$ of collision kernels that satisfy the following properties [Vil98]

\section{Definition 1 (The grazing sequence)}

The sequence $\left\{b_{\varepsilon}(\theta)\right\}$ is a grazing collision sequence if

(a) $b_{\varepsilon}(\theta)=b_{\varepsilon}(|\theta|) \geq 0$;

(b) $\operatorname{supp}\left\{b_{\varepsilon}\right\} \subset\left\{\theta \in\left[-\frac{\pi}{2}, \frac{\pi}{2}\right]: 0<c_{\varepsilon} \leq|\theta| \leq d_{\epsilon}\right\}$ where $d_{\varepsilon} \rightarrow 0$ for $\varepsilon \rightarrow 0$;

(c) $\int_{0}^{\frac{\pi}{2}} b_{\varepsilon}(\theta) \sin ^{2} \theta \mathrm{d} \theta=1$ for all $\varepsilon$.

The physical meaning of condition (ㄷ) is related to the fact that the cooling of the dissipative gas has to be guaranteed in the limit. By means of (4), it follows in fact that the energy varies in time according to

$$
\begin{aligned}
\frac{\mathrm{d}}{\mathrm{d} t} \int_{\mathbb{R}} v^{2} f(v, t) \mathrm{d} v & =\frac{1}{2} \int_{\mathbb{R}^{2}} \int_{-\pi / 2}^{\pi / 2} b_{\varepsilon}(\theta)\left(\left(v_{p}^{*}\right)^{2}+\left(w_{p}^{*}\right)^{2}-v^{2}-w^{2}\right) f(v, t) f(w, t) \mathrm{d} \theta \mathrm{d} w \mathrm{~d} v \\
& =-\left(\int_{-\pi / 2}^{\pi / 2} b_{\varepsilon}(\theta)\left(1-|\sin \theta|^{2+2 p}-|\cos \theta|^{2+2 p}\right) \mathrm{d} \theta\right) \int_{\mathbb{R}} v^{2} f(v, t) \mathrm{d} v .
\end{aligned}
$$

Hence, the grazing limit procedure has to be chosen so that the (positive) coefficient

$$
L_{\varepsilon}=\int_{-\pi / 2}^{\pi / 2} b_{\varepsilon}(\theta)\left(1-|\sin \theta|^{2+2 p}-|\cos \theta|^{2+2 p}\right) \mathrm{d} \theta
$$

remains bounded in the limit. It is immediate to conclude that, for $0<p \leq 1$

$$
1-|\sin \theta|^{2+2 p}-|\cos \theta|^{2+2 p} \leq 1-|\sin \theta|^{4}-|\cos \theta|^{4}=2 \sin ^{2} \theta \cos ^{2} \theta .
$$


Moreover, a simple analysis of the behavior of the function

$$
\Phi(y)=1-y^{1+p}-(1-y)^{1+p}-c_{p} y(1-y), \quad 0 \leq y \leq 1
$$

shows that $\Phi(y)$ is non-negative at least whenever $c_{p} \leq p(1+p) 2^{1-p}$. Consequently, we have the bounds

$$
c_{p} \int_{-\pi / 2}^{\pi / 2} b_{\varepsilon}(\theta) \sin ^{2} \theta \cos ^{2} \theta \mathrm{d} \theta \leq L_{\varepsilon} \leq 2 \int_{-\pi / 2}^{\pi / 2} b_{\varepsilon}(\theta) \sin ^{2} \theta \cos ^{2} \theta \mathrm{d} \theta
$$

which imply, under condition (ㄷ) of Definition 1, that the decay of energy remains well-defined in the limit procedure.

In the case of the elastic Kac equation, the grazing collision asymptotic has been studied in [Tos98], resorting to the non cut-off formulation by Desvillettes [Des95], in which the kernel $b$ is assumed to be non integrable. For a large class of initial densities, essentially all initial densities with finite energy, the solution to the kinetic model has been proven in [Tos98] to converge towards the solution to the linear Fokker-Planck equation

$$
\frac{\partial f}{\partial t}=\frac{\partial^{2} f}{\partial v^{2}}+\frac{\partial}{\partial v}(v f)
$$

In this paper we will address a similar problem, namely the grazing collision asymptotic of the inelastic Kac model, in the case in which the kernel $b$ is an integrable function, the initial data possess infinite energy and lie in a suitable neighborhood of a Lévy distribution of type (8).

Our main result (Theorem 5) consists in proving that, in the inelastic regime characterized by $0<p \leq 1$, the solution to the inelastic Kac model, corresponding to this choice of initial values, converges towards the solution of a Fokker-Planck equation with a fractional diffusion

$$
\frac{\partial f}{\partial t}=2\left(\alpha D^{q} f+\frac{1}{q} \frac{\partial}{\partial v}(v f)\right), \quad q=\frac{2}{1+p},
$$

where the fractional derivative of order $q$ is defined for instance by the Fourier formula

$$
D^{q} f=\mathcal{F}^{-1}\left(-|\xi|^{q} \widehat{f}(\xi)\right)
$$

and $\mathcal{F}^{-1}$ stands for the inverse Fourier transform.

Fokker-Planck type equations with fractional diffusion appear in many physical contexts Cha98, BWM00, SBMW01, MFL02, and have been intensively studied both from the modeling and the qualitative point of view. Likewise, Lévy distributions have been applied to the description of many physical processes, including turbulent flows [SWK87, diffusion in complex systems [OBLU90], chaotic dynamics of classical conservative systems [SZK93, KZB85, and others.

Nevertheless, the connections between fractional diffusion equations and kinetic models of Boltzmann type have been analyzed only recently. In the framework of kinetic theory and asymptotic limits, some insight on this connection has been recently 
done by Mellet, Mischler and Mouhot [MMM09]. The starting point of their analysis was the derivation of diffusion-type equations from a linear Boltzmann equation describing the interactions of the particles with the surrounding medium. Despite the classical problem, in which the Maxwellian distribution of the background decays exponentially fast at infinity, in the case described in [MMM09, the Maxwellian distribution is heavy-tailed, and the resulting diffusion limit corresponds to a fractional diffusion.

\section{$2 \quad$ Preliminary results}

In this short section we resume the main results relative to the dissipative Kac equation (1). The largest part of these results have been obtained in [PT04], where the kernel $b$, like in the original Kac model [Kac59], was assumed constant. The analysis of [PT04], however, can be easily extended to integrable kernels.

Theorem 2 (Existence, uniqueness and conservation laws)

Let $0<p \leq 1$. Let the initial datum $f_{0} \geq 0$ satisfy the (normalization) assumptions

$$
\int_{\mathbb{R}} f_{0}(v) \mathrm{d} v=1, \quad \int_{\mathbb{R}} v f_{0}(v) \mathrm{d} v=0
$$

Then, the initial value problem for the dissipative Kac equation (11)-(2), with $b \in$ $L^{1}([-\pi / 2, \pi / 2])$, has a unique non negative solution $f \in C^{1}\left([0, \infty), L^{1}(\mathbb{R})\right)$. This solution satisfies for all $t>0$ :

$$
\int_{\mathbb{R}} f(v, t) \mathrm{d} v=1, \quad \int_{\mathbb{R}} v f(v, t) \mathrm{d} v=0 .
$$

In this existence result, the initial data are not supposed to satisfy $\int_{\mathbb{R}}|v| f_{0}(v) \mathrm{d} v<+\infty$ and so $\int_{\mathbb{R}} v f_{0}(v) \mathrm{d} v=0$ has to be interpreted as a principal value integral. The proof of this theorem follows along the same lines of the proof for the conservative Kac equation, which goes back to Morgenstern [Mor54, Mor55].

Let us now consider the Fourier formulation (55)-(66), and let us suppose that the kernel $b$ is an even function. Then, Equations (5) -(6) can be written as

$$
\partial_{t} \widehat{f}(\xi, t)=\int_{0}^{\frac{\pi}{2}} b(\theta)\left(\widehat{f}\left(\xi \cos ^{p+1} \theta, t\right)\left(\widehat{f}\left(\xi \sin ^{p+1} \theta, t\right)+\widehat{f}\left(-\xi \sin ^{p+1} \theta, t\right)\right)-2 \widehat{f}(\xi, t)\right) \mathrm{d} \theta .
$$

Due to the integrability of the collision kernel, we can split the collision integral to obtain

$$
\partial_{t} \widehat{f}(\xi, t)=\int_{0}^{\frac{\pi}{2}} b(\theta) \widehat{f}\left(\xi \cos ^{p+1} \theta, t\right)\left(\widehat{f}\left(\xi \sin ^{p+1} \theta, t\right)+\widehat{f}\left(-\xi \sin ^{p+1} \theta, t\right) \mathrm{d} \theta-\sigma \widehat{f}(\xi, t)\right.
$$

where

$$
\sigma=\int_{-\frac{\pi}{2}}^{\frac{\pi}{2}} b(\theta) \mathrm{d} \theta
$$


The (unique) solution $\widehat{f}(t)$ of Equation (14) can be explicitly written using the socalled Wild expansion, found by Wild in Wil51 and exploited extensively since then by Bobylev ([Bob88]) and many others. It reads

$$
\widehat{f}(\xi, t)=\mathrm{e}^{-\sigma t} \sum_{n=0}^{\infty} \varphi_{n}(\xi)\left(1-e^{-\sigma t}\right)^{n},
$$

where

$$
\begin{aligned}
& \varphi_{0}(\xi)=\widehat{f}_{0}(\xi), \\
& \varphi_{n+1}(\xi)=\frac{1}{n+1} \sum_{j=0}^{n} Q_{+}\left(\varphi_{j}, \varphi_{n-j}\right)(\xi)
\end{aligned}
$$

and

$$
\begin{aligned}
Q_{+}(\varphi, \psi)(\xi)=\frac{1}{2 \sigma} \int_{0}^{\frac{\pi}{2}} b(\theta)[\varphi( & \left.\xi \cos ^{p+1} \theta\right)\left(\psi\left(\xi \sin ^{p+1} \theta\right)+\psi\left(-\xi \sin ^{p+1} \theta\right)\right) \\
& \left.+\psi\left(\xi \cos ^{p+1} \theta\right)\left(\varphi\left(\xi \sin ^{p+1} \theta\right)+\varphi\left(-\xi \sin ^{p+1} \theta\right)\right)\right] \mathrm{d} \theta
\end{aligned}
$$

Wild expansion follows from an iteration formula based on the solution written by Duhamel's formula

$$
\widehat{f}(\xi, t)=e^{-\sigma t} \widehat{f_{0}}(\xi)+\sigma \int_{0}^{t} e^{-\sigma(t-s)} Q_{+}(\widehat{f}, \widehat{f})(\xi, s) \mathrm{d} s .
$$

For any initial density $\widehat{f}_{0}$ the solution $\widehat{f}(t)$ is the limit of the sequence

$$
\widehat{f}^{(n)}(\xi, t)=e^{-\sigma t} \widehat{f}_{0}(\xi)+\sigma \int_{0}^{t} e^{-\sigma(t-s)} Q_{+}\left(\widehat{f}^{(n-1)}, \widehat{f}^{(n-1)}\right)(\xi, s) \mathrm{d} \theta \mathrm{d} s
$$

with $\widehat{f}^{(0)}(\xi, t)=\widehat{f}_{0}(\xi)$. It should be noticed however that the term $\widehat{f}^{(n)}$ does not correspond in general to a partial sum of (15). We will exploit in this paper the representation through Wild sums since it better suits our goals.

In what follows, we will assume that the initial data $f_{0}$ are even functions. Therefore, the solution itself is even together with its Fourier transform and Equation (14) reads

$$
\partial_{t} \widehat{f}(\xi, t)=2 \int_{0}^{\frac{\pi}{2}} b(\theta)\left(\widehat{f}\left(\xi \cos ^{p+1} \theta, t\right) \widehat{f}\left(\xi \sin ^{p+1} \theta, t\right)-\widehat{f}(\xi, t)\right) \mathrm{d} \theta .
$$

This assumption allows to simplify many details of the forthcoming proofs. It has to be noted, however, that the results continue to hold with minor modifications for general initial data which have first momentum equal to zero, at the price of an increasing number of computations.

A further result in [PT04] is concerned with the large time behavior of solutions.

\section{Theorem 3 ([PT04])}

Let $0<p \leq 1$ and let $f(t)$ be the unique solution of the dissipative Kac equation (11)-(2), corresponding to the initial density $f_{0}$ satisfying the normalization conditions (13), and such that, for some $0<\delta \leq 2 p /(p+1)$

$$
\int_{\mathbb{R}}|v|^{\frac{2}{p+1}+\delta}\left|f_{0}(v)-M_{p}(v)\right| \mathrm{d} v<+\infty .
$$


Then,

$$
\lim _{t \rightarrow+\infty} \sup _{\xi \neq 0} \frac{\left|\widehat{f}(\xi, t)-\widehat{M}_{p}(\xi)\right|}{|\xi|^{\frac{2}{p+1}+\delta}}=0 .
$$

\section{Remark 4}

Condition (18), which is a condition on moments of the initial data, can be replaced in the proof of Theorem 3 by the weaker condition

$$
\sup _{\xi \neq 0} \frac{\left|\widehat{f}_{0}(\xi)-\widehat{M}_{p}(\xi)\right|}{|\xi|^{\frac{2}{p+1}+\delta}}<+\infty .
$$

\section{Convergence to the solution of the Fokker-Planck equa- tion}

In this section we state and prove our main result on the grazing collision limit of solutions of the dissipative Kac equation (11)-(2) towards the solution of a Fokker-Planck equation with fractional diffusion (11).

In order to show why the Fokker-Planck equation (111) is the result of the grazing procedure, the following computation on the steady state $\widehat{M}_{p}(\xi)=\exp \left\{-\alpha|\xi|^{2 /(1+p)}\right\}$ with $\alpha>0$ will be useful.

Since $\widehat{M}_{p}(\xi)$ satisfies the Kac equation (5)

$$
\partial_{t} \widehat{M}_{p}(\xi)=2 \int_{0}^{\frac{\pi}{2}} b_{\varepsilon}(\theta)\left(\widehat{M}_{p}\left(\xi \cos ^{p+1} \theta\right) \widehat{M}_{p}\left(\xi \sin ^{p+1} \theta\right)-\widehat{M}_{p}(\xi)\right) \mathrm{d} \theta
$$

Let us expand $\widehat{M}_{p}$ around the origin and around the point $\xi$ respectively. Then

$$
\begin{aligned}
& \widehat{M}_{p}\left(\xi \sin ^{p+1} \theta\right)=1-\alpha|\xi|^{2 /(p+1)} \sin ^{2} \theta+o\left(|\xi|^{2 /(p+1)} \sin ^{2} \theta\right), \\
& \widehat{M}_{p}\left(\xi \cos ^{p+1} \theta\right)=\widehat{M}_{p}(\xi)+\partial_{\xi} \widehat{M}_{p}(\xi) \xi\left(\cos ^{p+1} \theta-1\right)+o\left(\xi\left(\cos ^{p+1} \theta-1\right)\right) .
\end{aligned}
$$

Using these expressions we obtain

$\partial_{t} \widehat{M}_{p}(\xi)=2 \int_{0}^{\frac{\pi}{2}} b_{\varepsilon}(\theta)\left(-\alpha|\xi|^{2 /(p+1)} \widehat{M}_{p}(\xi) \sin ^{2} \theta+\partial_{\xi} \widehat{M}_{p}(\xi) \xi\left(\cos ^{p+1} \theta-1\right)+R(\theta, \xi)\right) \mathrm{d} \theta$.

The reminder term $R(\theta, \xi)$ behaves like $\sin ^{2+\mu} \theta$ for $\mu>0$ when $\theta \rightarrow 0$. Now, the grazing conditions of Definition 1 imply $\int b_{\varepsilon}(\theta) \sin ^{2+\mu} \theta \mathrm{d} \theta \rightarrow 0$ as $\varepsilon \rightarrow 0$ and passing to the limit in $\varepsilon$ we get (at least in a formal way)

$$
\partial_{t} \widehat{M}_{p}(\xi)=-2 \alpha|\xi|^{2 /(p+1)} \widehat{M}_{p}(\xi)-(p+1) \xi \partial_{\xi} \widehat{M}_{p}(\xi),
$$

which is exactly the Fokker-Planck equation (11) in the Fourier variable. Our goal is to make this computation rigourous for any solution with initial data suitably close to the steady state $M_{p}$. 
It is easy to see, as it was done in CT98 for the classical Fokker-Planck equation, that the solution $f(t)$ of Equation (11) has an explicit expression in terms of a convolution between the initial data and the stationary state. In the Fourier variable this solution reads

$$
\widehat{f}(\xi, t)=\widehat{f}_{0}\left(\xi e^{-(p+1) t}\right) e^{-\alpha|\xi|^{\frac{2}{p+1}}\left(1-e^{-2 t}\right)} .
$$

In the physical space

$$
f(v, t)=\frac{1}{\beta(t)} f_{0}\left(\frac{\cdot}{\beta(t)}\right) * \frac{1}{\gamma(t)} M_{p}\left(\frac{\cdot}{\gamma(t)}\right)(v),
$$

with

$$
\beta(t)=e^{-(p+1) t}, \quad \gamma(t)=\left(1-e^{-2 t}\right)^{\frac{p+1}{2}}
$$

Note that

$$
\beta(t)^{2 /(p+1)}+\gamma(t)^{2 /(p+1)}=1
$$

\section{Theorem 5}

Assume $\left\{b_{\varepsilon}(\theta)\right\}_{\varepsilon>0} \subseteq L^{1}\left(\left[-\frac{\pi}{2}, \frac{\pi}{2}\right]\right)$ be a family of collision kernels satisfying Definition 1. Let $0<p \leq 1$ and let $f_{\varepsilon}(t) \in C^{1}\left([0,+\infty), L^{1}(\mathbb{R})\right)$ be the solutions of the dissipative Kac equations

$$
\partial_{t} \widehat{f}_{\varepsilon}(\xi, t)=2 \int_{0}^{\frac{\pi}{2}} b_{\varepsilon}(\theta)\left(\widehat{f}_{\varepsilon}\left(\xi \cos ^{p+1} \theta, t\right) \widehat{f}_{\varepsilon}\left(\xi \sin ^{p+1} \theta, t\right)-\widehat{f}_{\varepsilon}(\xi, t)\right) \mathrm{d} \theta
$$

corresponding to an even initial density $f_{0} \geq 0$ satisfying the normalization condition $\int f_{0}(v) \mathrm{d} v=1$.

Let us suppose in addition that the initial datum $f_{0}$ satisfies the conditions

A) there is $\alpha>0$ such that

$$
\lim _{\xi \rightarrow 0^{+}} \frac{1-\widehat{f_{0}}(\xi)}{\xi^{\frac{2}{p+1}}}=\alpha ;
$$

B) $\widehat{f}_{0}$ is differentiable outside the origin and the function $\partial_{\xi} \widehat{f}_{0}(\xi) / \xi^{\frac{1-p}{p+1}}$ is uniformly $\delta$-Hölder continuous on bounded subsets of $(0,+\infty)$, namely there is $\delta \in(0,1)$ and for all $R>0$ there is $K(R)>0$ such that

$$
\sup _{0<\xi \leq R, 0<\tau \leq R, \xi \neq \tau} \frac{\left|\frac{\partial_{\xi} \widehat{f}_{0}(\xi)}{\xi^{\frac{1-p}{p+1}}}-\frac{\partial_{\xi} \widehat{f}_{0}(\tau)}{\tau^{\frac{1-p}{p+1}}}\right|}{|\xi-\tau|^{\delta}} \leq K(R) .
$$

Then, if $f(t)$ is the solution (19) of the Fokker-Planck equation

$$
\partial_{t} \widehat{f}(\xi, t)=-2 \alpha|\xi|^{\frac{2}{p+1}} \widehat{f}(\xi, t)-(p+1) \xi \partial_{\xi} \widehat{f}(\xi, t),
$$

corresponding to the same initial datum $f_{0}$,

$$
\lim _{\varepsilon \rightarrow 0} \sup _{t \geq 0, \xi \neq 0} \frac{\left|\widehat{f}_{\varepsilon}(\xi, t)-\widehat{f}(\xi, t)\right|}{|\xi|^{\frac{2}{p+1}}}=0 .
$$


We begin by proving that assumptions A) and B) on the initial data, at least for $\varepsilon$ small enough, are uniformly propagated along the solutions $f_{\varepsilon}(t)$ of the dissipative Kac equation. In fact we have

\section{Lemma 6}

Assume $\left\{b_{\varepsilon}(\theta)\right\}_{\varepsilon>0} \subseteq L^{1}\left(\left[-\frac{\pi}{2}, \frac{\pi}{2}\right]\right)$ be a family of collision kernels satisfying properties (回) and (b) of Definition 1. Assume $f_{0} \geq 0$ is an even function, satisfying the normalization condition $\int f_{0}(v) \mathrm{d} v=1$.

A) If there is $\alpha>0$ such that

$$
\lim _{\xi \rightarrow 0^{+}} \frac{1-\widehat{f_{0}}(\xi)}{\xi^{\frac{2}{p+1}}}=\alpha
$$

then the solutions $\widehat{f}_{\varepsilon}(t)$ of the Kac equations (5)-(6) with $f_{0}$ as initial datum satisfies the same property uniformly in time and in $\varepsilon$, i.e.

$$
\lim _{\xi \rightarrow 0^{+}} \frac{1-\widehat{f}_{\varepsilon}(\xi, t)}{\xi^{\frac{2}{p+1}}}=\alpha \quad \text { uniformly in } \varepsilon \text { and } t .
$$

B) If in addition $\widehat{f}_{0}$ is differentiable outside the origin and the function $\partial_{\xi} \widehat{f}_{0}(\xi) / \xi^{\frac{1-p}{p+1}}$ is uniformly $\delta$-Hölder continuous on bounded subsets of $(0,+\infty)$, so that there is $\delta \in(0,1)$ and for all $R>0$ there is $K(R)>0$ such that

$$
\sup _{\xi, \tau \in(0, R], \xi \neq \tau} \frac{\left|\frac{\partial_{\xi} \widehat{f}_{0}(\xi)}{\xi^{\frac{1-p}{p+1}}}-\frac{\partial_{\xi} \widehat{f}_{0}(\tau)}{\tau^{\frac{1-p}{p+1}}}\right|}{|\xi-\tau|^{\delta}} \leq K(R)
$$

then the same is true uniformly for $\widehat{f}_{\varepsilon}(t)$, namely there is $\tilde{K}(R) \geq K(R)$ such that

$$
\sup _{t \geq 0} \sup _{\xi, \tau \in(0, R], \xi \neq \tau} \frac{\left|\frac{\partial_{\xi} \widehat{f}_{\varepsilon}(\xi, t)}{\xi^{\frac{1-p}{p+1}}}-\frac{\partial_{\xi} \widehat{f}_{\varepsilon}(\tau, t)}{\tau^{\frac{1-p}{p+1}}}\right|}{|\xi-\tau|^{\delta}} \leq \tilde{K}(R), \quad \text { for } \varepsilon \text { small enough. }
$$

Proof of Lemma 6; Since the stationary solution $\widehat{M}_{p}(\xi)=e^{-\alpha|\xi|^{\frac{2}{p+1}}}$ satisfies (21) , for all $\eta>0$ there exists $\lambda>0$ such that

$$
\sup _{0<\xi<\lambda} \frac{\left|\widehat{M}_{p}(\xi)-\widehat{f}_{0}(\xi)\right|}{\xi^{\frac{2}{p+1}}}<\eta .
$$

Hence it is enough to prove that for all $\varepsilon>0$ and $t>0$

$$
\sup _{0<\xi<\lambda} \frac{\left|\widehat{M}_{p}(\xi)-\widehat{f}_{\varepsilon}(\xi, t)\right|}{\xi^{\frac{2}{p+1}}}<\eta \text {. }
$$


The unique solution $\widehat{f}_{\varepsilon}(\xi, t)$ can be expressed using the Wild expansion (cfr. equation (15)). In the particular case of even initial data we have

$$
\widehat{f}_{\varepsilon}(\xi, t)=\mathrm{e}^{-\sigma_{\varepsilon} t} \sum_{n=0}^{\infty} \varphi_{n}^{\varepsilon}(\xi)\left(1-e^{-\sigma_{\varepsilon} t}\right)^{n},
$$

where

$$
\begin{gathered}
\sigma_{\varepsilon}=2 \int_{0}^{\frac{\pi}{2}} b_{\varepsilon}(\theta) \mathrm{d} \theta, \\
\varphi_{0}^{\varepsilon}(\xi)=\widehat{f}_{0}(\xi), \\
\varphi_{n+1}^{\varepsilon}(\xi)=\frac{1}{n+1} \sum_{j=0}^{n} Q_{+}^{\varepsilon}\left(\varphi_{j}^{\varepsilon}, \varphi_{n-j}^{\varepsilon}\right)(\xi),
\end{gathered}
$$

and

$$
Q_{+}^{\varepsilon}(\varphi, \psi)(\xi)=\frac{1}{\sigma_{\varepsilon}} \int_{0}^{\frac{\pi}{2}} b_{\varepsilon}(\theta)\left[\varphi\left(\xi \cos ^{p+1} \theta\right) \psi\left(\xi \sin ^{p+1} \theta\right)+\psi\left(\xi \cos ^{p+1} \theta\right) \varphi\left(\xi \sin ^{p+1} \theta\right)\right] \mathrm{d} \theta .
$$

Since for all $t>0$

$$
\mathrm{e}^{-\sigma_{\varepsilon} t} \sum_{n=0}^{\infty}\left(1-e^{-\sigma_{\varepsilon} t}\right)^{n}=1,
$$

inequality (26) follows provided

$$
\sup _{0<\xi<\lambda} \frac{\left|\widehat{M}_{p}(\xi)-\varphi_{n}^{\varepsilon}(\xi)\right|}{\xi^{\frac{2}{p+1}}}<\eta
$$

uniformly in $n$. It is enough to prove that (27) holds for $\varphi_{1}^{\varepsilon}$. Then by a recursive argument (27) holds for any $n>1$. Since

$$
\widehat{M}_{p}(\xi)=\frac{2}{\sigma_{\varepsilon}} \int_{0}^{\frac{\pi}{2}} b_{\varepsilon}(\theta) \widehat{M}_{p}\left(\xi \cos ^{p+1} \theta\right) \widehat{M}_{p}\left(\xi \sin ^{p+1} \theta\right) \mathrm{d} \theta,
$$

we have

$$
\begin{aligned}
\varphi_{1}^{\varepsilon}(\xi)-\widehat{M}_{p}(\xi)= & \frac{2}{\sigma_{\varepsilon}} \int_{0}^{\frac{\pi}{2}} b_{\varepsilon}(\theta)\left[\widehat{f}_{0}\left(\xi \cos ^{p+1} \theta\right) \widehat{f}_{0}\left(\xi \sin ^{p+1} \theta\right)-\widehat{M}_{p}\left(\xi \cos ^{p+1} \theta\right) \widehat{M}_{p}\left(\xi \sin ^{p+1} \theta\right)\right] \mathrm{d} \theta \\
= & \frac{2}{\sigma_{\varepsilon}} \int_{0}^{\frac{\pi}{2}} b_{\varepsilon}(\theta)\left\{\left[\widehat{f}_{0}\left(\xi \cos ^{p+1} \theta\right)-\widehat{M}_{p}\left(\xi \cos ^{p+1} \theta\right)\right] \widehat{f}_{0}\left(\xi \sin ^{p+1} \theta\right)\right. \\
& \left.\quad+\left[\widehat{f}_{0}\left(\xi \sin ^{p+1} \theta\right)-\widehat{M}_{p}\left(\xi \sin ^{p+1} \theta\right)\right] \widehat{M}_{p}\left(\xi \cos ^{p+1} \theta\right)\right\} \mathrm{d} \theta .
\end{aligned}
$$

Using the property $\left|\widehat{f}_{0}(\xi)\right| \leq 1$, we obtain

$$
\begin{array}{r}
\frac{\left|\varphi_{1}^{\varepsilon}(\xi)-\widehat{M}_{p}(\xi)\right|}{\xi^{\frac{2}{p+1}}} \leq \frac{2}{\sigma_{\varepsilon}} \int_{0}^{\frac{\pi}{2}} b_{\varepsilon}(\theta)\left(\cos ^{2} \theta \frac{\left|\widehat{f}_{0}\left(\xi \cos ^{p+1} \theta\right)-\widehat{M}_{p}\left(\xi \cos ^{p+1} \theta\right)\right|}{\left(\xi \cos ^{p+1} \theta\right)^{\frac{2}{p+1}}}\right. \\
\left.+\sin ^{2} \theta \frac{\left|\widehat{f}_{0}\left(\xi \sin ^{p+1} \theta\right)-\widehat{M}_{p}\left(\xi \sin ^{p+1} \theta\right)\right|}{\left(\xi \sin ^{p+1} \theta\right)^{\frac{2}{p+1}}}\right) \mathrm{d} \theta .
\end{array}
$$


Finally, since $0<\xi \cos ^{p+1} \theta<\xi$ and $0<\xi \sin ^{p+1} \theta<\xi$, by (25)

$$
\sup _{0<\xi<\lambda} \frac{\left|\varphi_{1}^{\varepsilon}(\xi, t)-\widehat{M}_{p}(\xi)\right|}{\xi^{\frac{2}{p+1}}} \leq \frac{2 \lambda}{\sigma_{\varepsilon}} \int_{0}^{\frac{\pi}{2}} b_{\varepsilon}(\theta) \mathrm{d} \theta=\lambda .
$$

This concludes the proof of part $A$ ). To prove $B$ ) consider that conditions (21) and (23) on the initial data imply

$$
\lim _{\xi \rightarrow 0^{+}} \frac{\partial_{\xi} \widehat{f}_{0}(\xi)}{\xi^{\frac{1-p}{p+1}}}=-\frac{2 \alpha}{p+1} .
$$

Indeed, by (21) and Cauchy theorem, there is $\left\{\tilde{\xi}_{n}\right\}$ such that $\tilde{\xi}_{n} \rightarrow 0^{+}$for $n \rightarrow+\infty$ and

$$
\lim _{n \rightarrow+\infty} \frac{\partial_{\xi} \widehat{f}_{0}\left(\tilde{\xi}_{n}\right)}{\tilde{\xi}_{n}^{\frac{1-p}{p+1}}}=-\frac{2 \alpha}{p+1} .
$$

Together with the Hölder continuity outside the origin (23), this leads to (28). Therefore

$$
\left|\frac{\partial_{\xi} \widehat{f_{0}}(\xi)}{\xi^{\frac{1-p}{p+1}}}+\frac{2 \alpha}{p+1}\right| \leq K(R)|\xi|^{\delta}, \quad \xi \in(0, R],
$$

and this implies

$$
\sup _{0<\xi \leq R} \frac{\left|\partial_{\xi} \widehat{f_{0}}(\xi)\right|}{\xi^{\frac{1-p}{p+1}}} \leq \frac{2 \alpha}{p+1}+K(R) R^{\delta}:=K_{1}(R)>0 .
$$

First of all, we prove that condition (29) is uniformly propagated on any $\varphi_{n}^{\varepsilon}$. For this purpose, it is enough to prove it for $\varphi_{1}^{\varepsilon}$. Recall that

$$
\varphi_{1}^{\varepsilon}(\xi)=\frac{2}{\sigma_{\varepsilon}} \int_{0}^{\frac{\pi}{2}} b_{\varepsilon}(\theta) \widehat{f}_{0}\left(\xi \cos ^{p+1} \theta\right) \widehat{f}_{0}\left(\xi \sin ^{p+1} \theta\right) \mathrm{d} \theta,
$$

and denote

$$
F_{0}(\xi)=\frac{\partial_{\xi} \widehat{f_{0}}(\xi)}{\xi^{\frac{1-p}{p+1}}} .
$$

By (29) and Lebesgue theorem, $\partial_{\xi} \varphi_{1}^{\varepsilon}(\xi)$ exists for all $\xi \neq 0$ and

$$
\begin{aligned}
\partial_{\xi} \varphi_{1}^{\varepsilon}(\xi)=\frac{2}{\sigma_{\varepsilon}} \int_{0}^{\frac{\pi}{2}} b_{\varepsilon}(\theta)\left[\cos ^{p+1} \theta \partial_{\xi} \widehat{f}_{0}\left(\xi \cos ^{p+1} \theta\right) \widehat{f}_{0}\left(\xi \sin ^{p+1} \theta\right)\right. \\
\left.+\sin ^{p+1} \theta \partial_{\xi} \widehat{f}_{0}\left(\xi \sin ^{p+1} \theta\right) \widehat{f}_{0}\left(\xi \cos ^{p+1} \theta\right)\right] \mathrm{d} \theta
\end{aligned}
$$

So, for $0<\xi \leq R$ we have

$$
\begin{aligned}
\frac{\partial_{\xi} \varphi_{1}^{\varepsilon}(\xi)}{\xi^{\frac{1-p}{p+1}}}=\frac{2}{\sigma_{\varepsilon}} \int_{0}^{\frac{\pi}{2}} b_{\varepsilon}(\theta)\left[\cos ^{2} \theta F_{0}\left(\xi \cos ^{p+1} \theta\right) \widehat{f}_{0}\left(\xi \sin ^{p+1} \theta\right)\right. & \\
& \left.+\sin ^{2} \theta F_{0}\left(\xi \sin ^{p+1} \theta\right) \widehat{f}_{0}\left(\xi \cos ^{p+1} \theta\right)\right] \mathrm{d} \theta
\end{aligned}
$$


Now, using (29) and the property $\left|\widehat{f}_{0}(\xi)\right| \leq 1$ we get

$$
\frac{\left|\partial_{\xi} \varphi_{1}^{\varepsilon}(\xi)\right|}{\xi^{\frac{1-p}{p+1}}} \leq \frac{2 K_{1}(R)}{\sigma_{\varepsilon}} \int_{0}^{\frac{\pi}{2}} b_{\varepsilon}(\theta) \mathrm{d} \theta=K_{1}(R) .
$$

By a recursive procedure, we get therefore for all $n>1$ and $0<\xi \leq R$

$$
\frac{\left|\partial_{\xi} \varphi_{n}^{\varepsilon}(\xi)\right|}{\xi^{\frac{1-p}{p+1}}} \leq K_{1}(R)
$$

and through Wild expansion (15), we get for all $t \geq 0$ and $0<\xi \leq R$

$$
\frac{\left|\partial_{\xi} \widehat{f}_{\varepsilon}(\xi, t)\right|}{\xi^{\frac{1-p}{p+1}}} \leq K_{1}(R) .
$$

Let us come now to the proof of (24) and, as we did before, we recover the result for the first term $\varphi_{1}^{\varepsilon}$. We have to prove that there is $\tilde{K}(R) \geq K(R)$ such that for $\xi, \tau \in(0, R]$ and at least for $\varepsilon$ small enough

$$
\left|\frac{\partial_{\xi} \varphi_{1}^{\varepsilon}(\xi)}{\xi^{\frac{1-p}{p+1}}}-\frac{\partial_{\xi} \varphi_{1}^{\varepsilon}(\tau)}{\tau^{\frac{1-p}{p+1}}}\right| \leq \tilde{K}(R)|\xi-\tau|^{\delta}
$$

where

$$
\begin{aligned}
& \frac{\partial_{\xi} \varphi_{1}^{\varepsilon}(\xi)}{\xi^{\frac{1-p}{p+1}}}-\frac{\partial_{\xi} \varphi_{1}^{\varepsilon}(\tau)}{\tau^{\frac{1-p}{p+1}}}= \\
& \frac{2}{\sigma_{\varepsilon}} \int_{0}^{\frac{\pi}{2}} b_{\varepsilon}(\theta)\left[\cos ^{2} \theta F_{0}\left(\xi \cos ^{p+1} \theta\right) \widehat{f}_{0}\left(\xi \sin ^{p+1} \theta\right)+\sin ^{2} \theta F_{0}\left(\xi \sin ^{p+1} \theta\right) \widehat{f}_{0}\left(\xi \cos ^{p+1} \theta\right)\right. \\
& \left.-\cos ^{2} \theta F_{0}\left(\tau \cos ^{p+1} \theta\right) \widehat{f}_{0}\left(\tau \sin ^{p+1} \theta\right)-\sin ^{2} \theta F_{0}\left(\tau \sin ^{p+1} \theta\right) \widehat{f}_{0}\left(\tau \cos ^{p+1} \theta\right)\right] \mathrm{d} \theta .
\end{aligned}
$$

Since $f_{0}$ satisfies (29), by Cauchy theorem we get for all $\xi$ and $\tau$ in $[0, R], \xi \neq \tau$

$$
\left|\frac{\widehat{f}_{0}(\xi)-\widehat{f}_{0}(\tau)}{\xi^{\frac{2}{p+1}}-\tau^{\frac{2}{p+1}}}\right|=\frac{p+1}{2}\left|\frac{\partial_{\xi} \widehat{f}_{0}(\bar{\xi})}{\bar{\xi}^{\frac{1-p}{p+1}}}\right| \leq \frac{p+1}{2} K_{1}(R):=K_{2}(R)
$$

with $\bar{\xi}$ between $\xi$ and $\tau$. Consequently $\widehat{f}_{0}$ satisfies

$$
\left|\widehat{f}_{0}(\xi)-\widehat{f}_{0}(\tau)\right| \leq K_{2}(R)\left|\xi^{\frac{2}{p+1}}-\tau^{\frac{2}{p+1}}\right| \quad \text { for all } \xi, \tau \in[0, R] .
$$


Using (23), (29) and (32), we get

$$
\begin{aligned}
& \left|\frac{\partial_{\xi} \varphi_{1}^{\varepsilon}(\xi)}{\xi^{\frac{1-p}{p+1}}}-\frac{\partial_{\xi} \varphi_{1}^{\varepsilon}(\tau)}{\tau^{\frac{1-p}{p+1}}}\right| \\
& \leq \frac{2}{\sigma_{\varepsilon}} \int_{0}^{\frac{\pi}{2}} b_{\varepsilon}(\theta)\left\{\operatorname { c o s } ^ { 2 } \theta \left[\left|F_{0}\left(\xi \cos ^{p+1} \theta\right)-F_{0}\left(\tau \cos ^{p+1} \theta\right)\right|\left|\widehat{f}_{0}\left(\xi \sin ^{p+1} \theta\right)\right|\right.\right. \\
& \left.+\left|F_{0}\left(\tau \cos ^{p+1} \theta\right)\right|\left|\widehat{f}_{0}\left(\xi \sin ^{p+1} \theta\right)-\widehat{f}_{0}\left(\tau \sin ^{p+1} \theta\right)\right|\right] \\
& +\sin ^{2} \theta\left[\left|F_{0}\left(\xi \sin ^{p+1} \theta\right)-F_{0}\left(\tau \sin ^{p+1} \theta\right)\right|\left|\widehat{f}_{0}\left(\xi \cos ^{p+1} \theta\right)\right|\right. \\
& \left.\left.+\left|F_{0}\left(\tau \sin ^{p+1} \theta\right)\right|\left|\widehat{f}_{0}\left(\xi \cos ^{p+1} \theta\right)-\widehat{f}_{0}\left(\tau \cos ^{p+1} \theta\right)\right|\right]\right\} \mathrm{d} \theta \\
& \leq \frac{2}{\sigma_{\varepsilon}} \int_{0}^{\frac{\pi}{2}} b_{\varepsilon}(\theta)\left\{\operatorname { c o s } ^ { 2 } \theta \left[K(R)\left|\xi \cos ^{p+1} \theta-\tau \cos ^{p+1} \theta\right|^{\delta}\right.\right. \\
& \left.+K_{2}(R) K_{1}(R)\left|\left(\xi \sin ^{p+1} \theta\right)^{\frac{2}{p+1}}-\left(\tau \sin ^{p+1} \theta\right)^{\frac{2}{p+1}}\right|\right] \\
& +\sin ^{2} \theta\left[K(R)\left|\xi \sin ^{p+1} \theta-\tau \sin ^{p+1} \theta\right|^{\delta}\right. \\
& \left.\left.+K_{2}(R) K_{1}(R)\left|\left(\xi \cos ^{p+1} \theta\right)^{\frac{2}{p+1}}-\left(\tau \cos ^{p+1} \theta\right)^{\frac{2}{p+1}}\right|\right]\right\} \mathrm{d} \theta \\
& \leq \frac{2}{\sigma_{\varepsilon}} \int_{0}^{\frac{\pi}{2}} b_{\varepsilon}(\theta)\left\{K(R)(\cos \theta)^{2+\delta(p+1)}|\xi-\tau|^{\delta}+K_{2}(R) K_{1}(R) \cos ^{2} \theta \sin ^{2} \theta\left|\xi^{\frac{2}{p+1}}-\tau^{\frac{2}{p+1}}\right|\right. \\
& \left.+K(R)(\sin \theta)^{2+\delta(p+1)}|\xi-\tau|^{\delta}+K_{2}(R) K_{1}(R) \sin ^{2} \theta \cos ^{2} \theta\left|\xi^{\frac{2}{p+1}}-\tau^{\frac{2}{p+1}}\right|\right\} \mathrm{d} \theta .
\end{aligned}
$$

Since

$$
\left|\xi^{\frac{2}{p+1}}-\tau^{\frac{2}{p+1}}\right|=\frac{2}{p+1}|\bar{\xi}|^{\frac{1-p}{p+1}}|\xi-\tau|,
$$

with $\bar{\xi}$ between $\xi$ and $\tau$, for $\xi, \tau \in(0, R], \delta \in(0,1)$ and $C(R)>0$ suitably chosen, we get

$$
\left|\xi^{\frac{2}{p+1}}-\tau^{\frac{2}{p+1}}\right| \leq C(R)|\xi-\tau|^{\delta} .
$$

It follows that

$$
\begin{aligned}
\left|\frac{\partial_{\xi} \varphi_{1}(\xi)}{\xi^{\frac{1-p}{p+1}}}-\frac{\partial_{\xi} \varphi_{1}(\tau)}{\tau^{\frac{1-p}{p+1}}}\right| \leq|\xi-\tau|^{\delta} \frac{2}{\sigma_{\varepsilon}} \int_{0}^{\frac{\pi}{2}} b_{\varepsilon}(\theta) & \left\{K(R)\left(\cos ^{2+\delta(p+1)} \theta+\sin ^{2+\delta(p+1)} \theta\right)\right. \\
& \left.+2 C(R) K_{2}(R) K_{1}(R) \cos ^{2} \theta \sin ^{2} \theta\right\} \mathrm{d} \theta .
\end{aligned}
$$

By assuming $\tilde{K}(R) \geq K(R)$ large enough, it is not restrictive to assume

$$
2 C(R) K_{2}(R) K_{1}(R) \leq \tilde{K}(R) .
$$

If this is the case,

$$
\begin{aligned}
& K(R)\left(\cos ^{2+\delta(p+1)} \theta+\sin ^{2+\delta(p+1)} \theta\right)+2 C(R) K_{2}(R) K_{1}(R) \cos ^{2} \theta \sin ^{2} \theta \\
& \leq \tilde{K}(R)\left(\cos ^{2+\delta(p+1)} \theta+\sin ^{2+\delta(p+1)} \theta+\cos ^{2} \theta \sin ^{2} \theta\right) .
\end{aligned}
$$


On the other hand, for $\theta$ sufficiently close to zero,

$$
\cos ^{2+\delta(p+1)} \theta+\sin ^{2+\delta(p+1)} \theta+\cos ^{2} \theta \sin ^{2} \theta=1-\frac{\delta(p+1)}{2} \theta^{2}+o\left(\theta^{2}\right) .
$$

Hence, there is $\bar{\theta}>0$ such that for $\theta \in[0, \bar{\theta}]$

$$
\cos ^{2+\delta(p+1)} \theta+\sin ^{2+\delta(p+1)} \theta+\cos ^{2} \theta \sin ^{2} \theta<1 .
$$

By condition (b) of Definition 1 for $\varepsilon$ small enough

$$
\begin{aligned}
& \left|\frac{\partial_{\xi} \varphi_{1}^{\varepsilon}(\xi)}{\xi^{\frac{1-p}{p+1}}}-\frac{\partial_{\xi} \varphi_{1}^{\varepsilon}(\tau)}{\tau^{\frac{1-p}{p+1}}}\right| \\
& \leq \tilde{K}(R)|\xi-\tau|^{\delta} \frac{2}{\sigma_{\varepsilon}} \int_{0}^{\bar{\theta}} b_{\varepsilon}(\theta)\left(\cos ^{2+\delta(p+1)} \theta+\sin ^{2+\delta(p+1)} \theta+\cos ^{2} \theta \sin ^{2} \theta\right) \mathrm{d} \theta \\
& \leq \tilde{K}(R)|\xi-\tau|^{\delta} \frac{2}{\sigma_{\varepsilon}} \int_{0}^{\bar{\theta}} b_{\varepsilon}(\theta) \mathrm{d} \theta \leq \tilde{K}(R)|\xi-\tau|^{\delta}
\end{aligned}
$$

Finally

$$
\left|\frac{\partial_{\xi} \varphi_{1}^{\varepsilon}(\xi)}{\xi^{\frac{1-p}{p+1}}}-\frac{\partial_{\xi} \varphi_{1}^{\varepsilon}(\tau)}{\tau^{\frac{1-p}{p+1}}}\right| \leq \tilde{K}(R)|\xi-\tau|^{\delta} .
$$

Recursively, we can prove the same estimate for $\varphi_{n}^{\varepsilon}, n>1$ and therefore for the solution $\widehat{f}_{\varepsilon}$. This concludes the proof of part $B$ ).

We are now in a position to prove Theorem 5.

Proof of Theorem 5: Let us first underline again that if $f_{0}$ is even, then the solutions $f_{\varepsilon}(t)$ and $f(t)$ are even functions together with their Fourier transforms. Our goal will be to prove that

$$
\lim _{\varepsilon \rightarrow 0} \sup _{t \geq 0, \xi \neq 0} \frac{\left|\widehat{f}_{\varepsilon}(\xi, t)-\widehat{f}(\xi, t)\right|}{|\xi|^{\frac{2}{p+1}}}=0 .
$$

In more detail, we will show that for all $\lambda>0$ there is $\bar{\varepsilon}=\bar{\varepsilon}(\lambda)$ such that for all $0<\varepsilon<\bar{\varepsilon}$

$$
\sup _{t \geq 0, \xi \neq 0} \frac{\left|\widehat{f}_{\varepsilon}(\xi, t)-\widehat{f}(\xi, t)\right|}{|\xi|^{\frac{2}{p+1}}}<\lambda .
$$

Since $\left|\widehat{f}_{\varepsilon}(\xi, t)-\widehat{f}(\xi, t)\right| \leq 2$, (34) holds for $\xi \geq R>0$, if $R$ is large enough (it is enough to let $R>\left(\frac{2}{\lambda}\right)^{\frac{p+1}{2}}$ ). Therefore, let us prove (134) when $0<|\xi| \leq R$. Since $\widehat{f_{\varepsilon}}$ and $\widehat{f}$ are even functions, it is enough to consider $\xi>0$. It holds

$$
\begin{array}{r}
\partial_{t}\left(\widehat{f}_{\varepsilon}(\xi, t)-\widehat{f}(\xi, t)\right)=2 \int_{0}^{\frac{\pi}{2}} b_{\varepsilon}(\theta)\left(\widehat{f}_{\varepsilon}\left(\xi \cos ^{p+1} \theta, t\right) \widehat{f}_{\varepsilon}\left(\xi \sin ^{p+1} \theta, t\right)-\widehat{f}_{\varepsilon}(\xi, t)\right) \mathrm{d} \theta \\
+2 \alpha \xi^{\frac{2}{p+1}} \widehat{f}(\xi, t)+(p+1) \xi \partial_{\xi} \widehat{f}(\xi, t)
\end{array}
$$


In what follows, in order to shorten formulas, we will often drop the dependence of the $t$ variable. We get

$$
\begin{aligned}
\partial_{t}\left(\widehat{f}_{\varepsilon}(\xi)-\widehat{f}(\xi)\right) & =-2 \alpha \xi^{\frac{2}{p+1}}\left(\widehat{f}_{\varepsilon}(\xi)-\widehat{f}(\xi)\right)-(p+1) \xi \partial_{\xi}\left(\widehat{f}_{\varepsilon}(\xi)-\widehat{f}(\xi)\right) \\
+2 \int_{0}^{\frac{\pi}{2}} b_{\varepsilon}(\theta)\left(\widehat{f}_{\varepsilon}\left(\xi \cos ^{p+1} \theta\right) \widehat{f}_{\varepsilon}\left(\xi \sin ^{p+1} \theta\right)-\widehat{f}_{\varepsilon}(\xi)\right) \mathrm{d} \theta & +2 \alpha \xi^{\frac{2}{p+1}} \widehat{f}_{\varepsilon}(\xi)+(p+1) \xi \partial_{\xi} \widehat{f}_{\varepsilon}(\xi),
\end{aligned}
$$

that corresponds to

$$
\partial_{t}\left(\frac{\widehat{f}_{\varepsilon}(\xi)-\widehat{f}(\xi)}{\xi^{\frac{2}{p+1}}}\right)=-2 \alpha\left(\widehat{f}_{\varepsilon}(\xi)-\widehat{f}(\xi)\right)-(p+1) \frac{\partial_{\xi}\left(\widehat{f}_{\varepsilon}(\xi)-\widehat{f}(\xi)\right)}{\xi^{\frac{1-p}{p+1}}}+R_{\varepsilon}(\xi, t)
$$

where

$$
\begin{aligned}
& R_{\varepsilon}(\xi, t)=\frac{2}{\xi^{\frac{2}{p+1}}} \int_{0}^{\frac{\pi}{2}} b_{\varepsilon}(\theta) \widehat{f}_{\varepsilon}\left(\xi \cos ^{p+1} \theta\right)\left(\widehat{f}_{\varepsilon}\left(\xi \sin ^{p+1} \theta\right)-1\right) \mathrm{d} \theta+2 \alpha \widehat{f}_{\varepsilon}(\xi) \\
& +\frac{2}{\xi^{\frac{2}{p+1}}} \int_{0}^{\frac{\pi}{2}} b_{\varepsilon}(\theta)\left(\widehat{f}_{\varepsilon}\left(\xi \cos ^{p+1} \theta\right)-\widehat{f}_{\varepsilon}(\xi)\right) \mathrm{d} \theta+(p+1) \frac{\partial_{\xi} \widehat{f}_{\varepsilon}(\xi)}{\xi^{\frac{1-p}{p+1}}}=A_{\varepsilon}(\xi, t)+B_{\varepsilon}(\xi, t) .
\end{aligned}
$$

Thanks to assumption (ㄷ) on $b_{\varepsilon}$ we obtain

$$
\begin{aligned}
A_{\varepsilon}(\xi, t)= & \frac{2}{\xi^{\frac{2}{p+1}}} \int_{0}^{\frac{\pi}{2}} b_{\varepsilon}(\theta) \widehat{f}_{\varepsilon}\left(\xi \cos ^{p+1} \theta\right)\left(\widehat{f}_{\varepsilon}\left(\xi \sin ^{p+1} \theta\right)-1\right) \mathrm{d} \theta+2 \alpha \widehat{f}_{\varepsilon}(\xi) \\
= & 2 \int_{0}^{\frac{\pi}{2}} b_{\varepsilon}(\theta) \sin ^{2} \theta \widehat{f}_{\varepsilon}\left(\xi \cos ^{p+1} \theta\right) \frac{\widehat{f}_{\varepsilon}\left(\xi \sin ^{p+1} \theta\right)-1}{\left(\xi \sin ^{p+1} \theta\right)^{\frac{2}{p+1}}} \mathrm{~d} \theta+2 \alpha \widehat{f}_{\varepsilon}(\xi) \\
= & 2 \int_{0}^{\frac{\pi}{2}} b_{\varepsilon}(\theta) \sin ^{2} \theta \widehat{f}_{\varepsilon}\left(\xi \cos ^{p+1} \theta\right)\left(\frac{\widehat{f}_{\varepsilon}\left(\xi \sin ^{p+1} \theta\right)-1}{\left(\xi \sin ^{p+1} \theta\right)^{\frac{2}{p+1}}}+\alpha\right) \mathrm{d} \theta \\
& \quad-2 \alpha \int_{0}^{\frac{\pi}{2}} b_{\varepsilon}(\theta) \sin ^{2} \theta\left(\widehat{f}_{\varepsilon}\left(\xi \cos ^{p+1} \theta\right)-\widehat{f}_{\varepsilon}(\xi)\right) \mathrm{d} \theta=A_{1, \varepsilon}(\xi, t)+A_{2, \varepsilon}(\xi, t) .
\end{aligned}
$$

Let us estimate first $A_{1, \varepsilon}$. Since $\left\|\widehat{f}_{\varepsilon}\right\|_{\infty} \leq 1$, by assumptions (b) and (ㄷ) on $b_{\varepsilon}$ we get for all $t \geq 0$ and $0<\xi \leq R$

$$
\begin{array}{r}
\left|A_{1, \varepsilon}(\xi, t)\right| \leq 2 \sup _{t \geq 0,} c_{\varepsilon} \leq \theta \leq d_{\varepsilon}, 0<\xi \leq R \\
\quad \leq 2 \sup _{t \geq 0,0<\eta \leq R\left(\sin d_{\varepsilon}\right)^{p+1}}\left|\frac{\widehat{f}_{\varepsilon}\left(\xi \sin ^{p+1} \theta, t\right)-1}{\left(\xi \sin ^{p+1} \theta\right)^{\frac{2}{p+1}}}+\alpha\right| \\
\eta^{\frac{2}{p+1}}+\alpha \mid
\end{array}
$$

Now, remembering the uniform convergence in condition (22), we get

$$
\sup _{t \geq 0,0<\xi \leq R}\left|A_{1, \varepsilon}(\xi, t)\right|=A(\varepsilon) \rightarrow 0, \quad \varepsilon \rightarrow 0 .
$$


Let us come to $A_{2, \varepsilon}$. By Lagrange theorem, for all $t \geq 0$ and $0<\xi \leq R$ we obtain

$$
\left|A_{2, \varepsilon}(\xi, t)\right| \leq 2 \alpha \int_{0}^{\frac{\pi}{2}} b_{\varepsilon}(\theta) \sin ^{2} \theta\left|\partial_{\xi} \widehat{f}_{\varepsilon}(\bar{\xi}(\varepsilon, \xi, t))\right| \xi\left|\cos ^{p+1} \theta-1\right| \mathrm{d} \theta
$$

where $0<\xi \cos ^{p+1} \theta<\bar{\xi}(\varepsilon, \xi, t)<\xi \leq R$.

Thanks to (31), for $\varepsilon$ small enough

$$
\sup _{t \geq 0,0<\xi \leq R}\left|\partial_{\xi} \widehat{f}_{\varepsilon}(\xi, t)\right| \leq R^{\frac{1-p}{p+1}} K_{1}(R):=C^{\prime}(R)
$$

and by assumption (Bb) on the support of $b_{\varepsilon}\left|\cos ^{p+1} \theta-1\right| \leq 1-\left(\cos d_{\varepsilon}\right)^{p+1} \rightarrow 0$ for $\varepsilon \rightarrow 0$. Finally, owing to assumption (ㄷ) on $b_{\varepsilon}$ we obtain

$$
\sup _{t \geq 0,0<\xi \leq R}\left|A_{2, \varepsilon}(\xi, t)\right| \leq 2 \alpha C^{\prime}(R) R\left(1-\left(\cos d_{\varepsilon}\right)^{p+1}\right)=B(\varepsilon) \rightarrow 0, \quad \varepsilon \rightarrow 0 .
$$

Let us now pass to estimate the term $B_{\varepsilon}$ in (36). By assumption (드) on $b_{\varepsilon}$ we get

$$
\begin{aligned}
B_{\varepsilon}(\xi, t) & =2 \int_{0}^{\frac{\pi}{2}} b_{\varepsilon}(\theta) \frac{\widehat{f}_{\varepsilon}\left(\xi \cos ^{p+1} \theta\right)-\widehat{f}_{\varepsilon}(\xi)}{\xi^{\frac{2}{p+1}}} \mathrm{~d} \theta+(p+1) \frac{\partial_{\xi} \widehat{f}_{\varepsilon}(\xi)}{\xi^{\frac{1-p}{p+1}}} \\
& =-2 \int_{0}^{\frac{\pi}{2}} b_{\varepsilon}(\theta) \sin ^{2} \theta\left(\frac{\widehat{f}_{\varepsilon}\left(\xi \cos ^{p+1} \theta\right)-\widehat{f}_{\varepsilon}(\xi)}{\left(\xi \cos ^{p+1} \theta\right)^{\frac{2}{p+1}}-\xi^{\frac{2}{p+1}}}-\frac{p+1}{2} \frac{\partial_{\xi} \widehat{f}_{\varepsilon}(\xi)}{\xi^{\frac{1-p}{p+1}}}\right) \mathrm{d} \theta .
\end{aligned}
$$

By Cauchy theorem, for all $t \geq 0$ and $0<\xi \leq R$ we obtain

$$
\left|B_{\varepsilon}(\xi, t)\right| \leq(p+1) \int_{0}^{\frac{\pi}{2}} b_{\varepsilon}(\theta) \sin ^{2} \theta\left|\frac{\partial_{\xi} \widehat{f}_{\varepsilon}(\tilde{\xi})}{\tilde{\xi}^{\frac{1-p}{p+1}}}-\frac{\partial_{\xi} \widehat{f}_{\varepsilon}(\xi)}{\xi^{\frac{1-p}{p+1}}}\right| \mathrm{d} \theta
$$

where $\tilde{\xi}$ depends on $\varepsilon, \theta, \xi, t$ and lies in $\left(\xi \cos ^{p+1} \theta, \xi\right) \subset(0, R)$. Thanks to the uniform Hölder continuity (24)

$$
(p+1) \int_{0}^{\frac{\pi}{2}} b_{\varepsilon}(\theta) \sin ^{2} \theta\left|\frac{\partial_{\xi} \widehat{f}_{\varepsilon}(\tilde{\xi})}{\tilde{\xi}^{\frac{1-p}{p+1}}}-\frac{\partial_{\xi} \widehat{f}_{\varepsilon}(\xi)}{\xi^{\frac{1-p}{p+1}}}\right| \mathrm{d} \theta \leq(p+1) \tilde{K}(R) \int_{0}^{\frac{\pi}{2}} b_{\varepsilon}(\theta) \sin ^{2} \theta|\tilde{\xi}-\xi|^{\delta} \mathrm{d} \theta,
$$

and by condition (b),$|\tilde{\xi}-\xi|^{\delta}<|\xi|^{\delta}\left(1-\cos ^{p+1} \theta\right)^{\delta} \leq R^{\delta}\left(1-\left(\cos d_{\varepsilon}\right)^{p+1}\right)^{\delta} \rightarrow 0$ as $\varepsilon \rightarrow 0$. Therefore

$$
\sup _{t \geq 0,0<\xi \leq R}\left|B_{\varepsilon}(\xi, t)\right| \leq(p+1) \tilde{K}(R) R^{\delta}\left(1-\left(\cos d_{\varepsilon}\right)^{p+1}\right)^{\delta}=C(\varepsilon) \rightarrow 0, \quad \varepsilon \rightarrow 0 .
$$

Going back to equation (35), we proved that

$$
\sup _{t \geq 0,0<\xi \leq R}\left|R_{\varepsilon}(\xi, t)\right|=A(\varepsilon)+B(\varepsilon)+C(\varepsilon) \rightarrow 0, \quad \varepsilon \rightarrow 0 .
$$

Moreover, since

$$
\partial_{\xi}\left(\frac{\widehat{f}_{\varepsilon}(\xi)-\widehat{f}(\xi)}{\xi^{\frac{2}{p+1}}}\right)=\frac{\partial_{\xi}\left(\widehat{f}_{\varepsilon}(\xi)-\widehat{f}(\xi)\right)}{\xi^{\frac{2}{p+1}}}-\frac{2}{p+1} \frac{\widehat{f}_{\varepsilon}(\xi)-\widehat{f}(\xi)}{\xi^{\frac{2}{p+1}+1}}
$$


we obtain

$$
\frac{\partial_{\xi}\left(\widehat{f}_{\varepsilon}(\xi)-\widehat{f}(\xi)\right)}{\xi^{\frac{1-p}{p+1}}}=\xi \partial_{\xi}\left(\frac{\widehat{f}_{\varepsilon}(\xi)-\widehat{f}(\xi)}{\xi^{\frac{2}{p+1}}}\right)+\frac{2}{p+1} \frac{\widehat{f}_{\varepsilon}(\xi)-\widehat{f}(\xi)}{\xi^{\frac{2}{p+1}}}
$$

so that (35) can be written as

$$
\begin{aligned}
\partial_{t}\left(\frac{\widehat{f}_{\varepsilon}(\xi)-\widehat{f}(\xi)}{\xi^{\frac{2}{p+1}}}\right)=-2 \alpha \xi^{\frac{2}{p+1}}\left(\frac{\widehat{f}_{\varepsilon}(\xi)-\widehat{f}(\xi)}{\xi^{\frac{2}{p+1}}}\right)- & (p+1) \xi \partial_{\xi}\left(\frac{\widehat{f_{\varepsilon}}(\xi)-\widehat{f}(\xi)}{\xi^{\frac{2}{p+1}}}\right) \\
& -2\left(\frac{\widehat{f}_{\varepsilon}(\xi)-\widehat{f}(\xi)}{\xi^{\frac{2}{p+1}}}\right)+R_{\varepsilon}(\xi, t) .
\end{aligned}
$$

Let us denote

$$
y_{\varepsilon}(\xi, t)=\frac{\widehat{f_{\varepsilon}}(\xi, t)-\widehat{f}(\xi, t)}{\xi^{\frac{2}{p+1}}} .
$$

With this definition we obtained

$$
\partial_{t} y_{\varepsilon}(\xi, t)+(p+1) \xi \partial_{\xi} y_{\varepsilon}(\xi, t)=-2\left(\alpha \xi^{\frac{2}{p+1}}+1\right) y_{\varepsilon}(\xi, t)+R_{\varepsilon}(\xi, t) .
$$

Let us further define

$$
z_{\varepsilon}(\xi, t):=y_{\varepsilon}\left(\xi e^{(p+1) t}, t\right)
$$

Then

$$
\partial_{t} z_{\varepsilon}(\xi, t)=(p+1) \xi e^{(p+1) t} \partial_{\xi} y_{\varepsilon}\left(\xi e^{(p+1) t}, t\right)+\partial_{t} y_{\varepsilon}\left(\xi e^{(p+1) t}, t\right),
$$

and $z_{\varepsilon}$ satisfies

$$
\partial_{t} z_{\varepsilon}(\xi, t)=-2\left(\alpha \xi^{\frac{2}{p+1}} e^{2 t}+1\right) z_{\varepsilon}(\xi, t)+R_{\varepsilon}\left(\xi e^{(p+1) t}, t\right) .
$$

Integrating in time we get

$$
\begin{aligned}
z_{\varepsilon}(\xi, t)=z_{\varepsilon}(\xi, 0) & e^{-\left(\alpha \xi^{\frac{2}{p+1}}\left(e^{2 t}-1\right)+2 t\right)} \\
& +e^{-\left(\alpha \xi^{\frac{2}{p+1}}\left(e^{2 t}-1\right)+2 t\right)} \int_{0}^{t} R_{\varepsilon}\left(\xi e^{(p+1) s}, s\right) e^{\left(\alpha \xi^{\frac{2}{p+1}}\left(e^{2 s}-1\right)+2 s\right)} \mathrm{d} s .
\end{aligned}
$$

Since $\widehat{f}_{\varepsilon}(t)$ and $\widehat{f}(t)$ correspond to the same initial data, $z_{\varepsilon}(\xi, 0)=y_{\varepsilon}(\xi, 0)=0$. From (39) we obtain

$$
y_{\varepsilon}(\xi, t)=e^{-\left(\alpha \xi^{\frac{2}{p+1}}\left(1-e^{-2 t}\right)+2 t\right)} \int_{0}^{t} R_{\varepsilon}\left(\xi e^{-(p+1)(t-s)}, s\right) e^{\left(\alpha \xi^{\frac{2}{p+1}} e^{-2 t}\left(e^{2 s}-1\right)+2 s\right)} \mathrm{d} s .
$$

Hence, for all $t \geq 0$ and $0<\xi \leq R$

$$
\begin{aligned}
\left|y_{\varepsilon}(\xi, t)\right| & \leq e^{-\left(\alpha \xi^{\frac{2}{p+1}}\left(1-e^{-2 t}\right)+2 t\right)} \int_{0}^{t}\left|R_{\varepsilon}\left(\xi e^{-(p+1)(t-s)}, s\right)\right| e^{\left(\alpha \xi^{\frac{2}{p+1}} e^{-2 t}\left(e^{2 t}-1\right)+2 s\right)} \mathrm{d} s \\
& \leq \sup _{t \geq 0,0<\eta \leq R}\left|R_{\varepsilon}(\eta, t)\right| e^{-2 t} \int_{0}^{t} e^{2 s} \mathrm{~d} s=(A(\varepsilon)+B(\varepsilon)+C(\varepsilon)) \frac{e^{-2 t}}{2}\left(e^{2 t}-1\right) \\
& \leq \frac{1}{2}(A(\varepsilon)+B(\varepsilon)+C(\varepsilon)) .
\end{aligned}
$$


Finally, for all $\lambda>0$ we proved that there is $\bar{\varepsilon}=\bar{\varepsilon}(\lambda)$ such that for all $0<\varepsilon<\bar{\varepsilon}$

$$
\sup _{t \geq 0,0<\xi \leq R} \frac{\left|\widehat{f}_{\varepsilon}(\xi, t)-\widehat{f}(\xi, t)\right|}{\xi^{\frac{2}{p+1}}}<\lambda .
$$

This ends the proof.

In order to give a simple example of initial data which fulfill the assumptions made in Theorem 5 , it is enough to consider

$$
f_{0}(v)=\frac{1}{2}\left(\widetilde{M}_{p}+\varphi\right)(v)
$$

where $\widetilde{M}_{p}(v)=\mathcal{F}^{-1}\left(e^{-2 \alpha|\xi|^{\frac{2}{p+1}}}\right)$ and $\varphi$ is an even probability density which satisfies $\int|v|^{\frac{2}{p+1}+\delta} \varphi(v) \mathrm{d} v<+\infty$, for $0<\delta \leq \frac{2 p}{p+1}$. Note that an even probability density with bounded energy satisfies this assumption. In this case $\lim _{\xi \rightarrow 0^{+}} \frac{1-\widehat{f}_{0}(\xi)}{\xi^{\frac{2}{p+1}}}=\alpha$. Moreover $\frac{\partial_{\xi} \widehat{\bar{M}}_{p}(\xi)}{\xi^{\frac{1-p}{p+1}}}$ is uniformly $\delta$-Hölder continuous on bounded subsets of $(0,+\infty)$. On the other hand, thanks to the moment condition we can show that for $\frac{\partial_{\xi} \widehat{\varphi}(\xi)}{\xi^{\frac{1-p}{p+1}}}$ is uniformly $\delta$-Hölder continuous on $(0,+\infty)$. To this extent, since $\varphi$ is an even function we can write

$$
\frac{\partial_{\xi} \widehat{\varphi}(\xi)}{\xi^{\frac{1-p}{p+1}}}=-\int v \varphi(v) \frac{\sin (v \xi)}{\xi^{\frac{1-p}{p+1}}} \mathrm{~d} v .
$$

We remark that, for $x>0$, the function $\frac{\sin x}{x^{\frac{1-p}{p+1}}}$ is uniformly $\delta$-Hölder continuous for any $0<\delta \leq \frac{2 p}{p+1}$. In fact for $|x-y| \geq 1$

$$
\left|\frac{\sin x}{x^{\frac{1-p}{p+1}}}-\frac{\sin y}{y^{\frac{1-p}{p+1}}}\right| \leq C \leq C|x-y|^{\delta} .
$$

For $|x-y| \leq 1$ (and $0<x<y$ with no loss in generality), Cauchy theorem guarantees that there is $x<\bar{x}<y$ such that

$$
\begin{aligned}
& \left|\frac{\frac{\sin x}{x^{\frac{1-p}{p+1}}}-\frac{\sin y}{y^{\frac{1-p}{p+1}}}}{x^{\frac{2 p}{p+1}}-y^{\frac{2 p}{p+1}}}\right|=\left|\frac{\frac{\cos \bar{x}}{\bar{x}^{\frac{1-p}{p+1}}}-\frac{1-p}{p+1} \frac{\sin \bar{x}}{\bar{x}^{\frac{2}{p+1}}}}{\frac{2 p}{p+1} \bar{x}^{\frac{p-1}{1+p}}}\right| \\
& =\frac{p+1}{2 p}\left|\cos \bar{x}-\frac{1-p}{p+1} \frac{\sin \bar{x}}{\bar{x}}\right| \leq C .
\end{aligned}
$$

Therefore, for $0<\delta \leq \frac{2 p}{p+1}$

$$
\left|\frac{\sin x}{x^{\frac{1-p}{p+1}}}-\frac{\sin y}{y^{\frac{1-p}{p+1}}}\right| \leq C\left|x^{\frac{2 p}{p+1}}-y^{\frac{2 p}{p+1}}\right| \leq C|x-y|^{\frac{2 p}{p+1}} \leq C|x-y|^{\delta} .
$$


Finally, for $\xi, \tau>0$ we get

$$
\begin{aligned}
& \left|\frac{\partial_{\xi} \widehat{\varphi}(\xi)}{\xi^{\frac{1-p}{p+1}}}-\frac{\partial_{\tau} \widehat{\varphi}(\tau)}{\tau^{\frac{1-p}{p+1}}}\right| \leq \int|v| \varphi(v)\left|\frac{\sin (v \xi)}{\xi^{\frac{1-p}{p+1}}}-\frac{\sin (v \tau)}{\tau^{\frac{1-p}{p+1}}}\right| \mathrm{d} v \\
& =\int|v|^{\frac{2}{p+1}} \varphi(v)\left|\frac{\sin (v \xi)}{(|v| \xi)^{\frac{1-p}{p+1}}}-\frac{\sin (v \tau)}{(|v| \tau)^{\frac{1-p}{p+1}}}\right| \mathrm{d} v \\
& \leq C \int|v|^{\frac{2}{p+1}} \varphi(v)|| v|\xi-| v|\tau|^{\delta} \mathrm{d} v \\
& =C|\xi-\tau|^{\delta} \int|v|^{\frac{2}{p+1}+\delta} \varphi(v) \mathrm{d} v=C|\xi-\tau|^{\delta} .
\end{aligned}
$$

A second example is furnished by the initial datum

$$
f_{0}(v)=\left(M_{p} * \varphi\right)(v)
$$

where $M_{p}(v)=\mathcal{F}^{-1}\left(e^{-\alpha|\xi|^{\frac{2}{p+1}}}\right)$ and $\varphi$ is as above.

It is interesting to remark that, under the assumptions on the initial data given in Theorem 5], we obtain the large-time convergence result of [PT04].

\section{Proposition 7}

Let $0<p \leq 1$ and let $f_{\varepsilon}(t)$ the unique solution of the dissipative Kac equation

$$
\partial_{t} \widehat{f}_{\varepsilon}(\xi, t)=2 \int_{0}^{\frac{\pi}{2}} b_{\varepsilon}(\theta)\left(\widehat{f}_{\varepsilon}\left(\xi \cos ^{p+1} \theta, t\right) \widehat{f}_{\varepsilon}\left(\xi \sin ^{p+1} \theta, t\right)-\widehat{f}_{\varepsilon}(\xi, t)\right) \mathrm{d} \theta
$$

with even initial density $f_{0} \geq 0$ satisfying the normalization condition $\int f_{0}(v) \mathrm{d} v=1$ and assumptions (21) and (23) of Theorem [5. Let $\widehat{M}_{p}(\xi)=e^{-\alpha|\xi|^{\frac{2}{p+1}}}$ the stationary state with $\alpha$ as in condition (21). Then, for any $0<\delta^{\prime} \leq \delta$

$$
\lim _{t \rightarrow+\infty} \sup _{\xi \neq 0} \frac{\left|\widehat{f}_{\varepsilon}(\xi, t)-\widehat{M}_{p}(\xi)\right|}{|\xi|^{\frac{2}{p+1}+\delta^{\prime}}}=0 .
$$

Proof: As already remarked, it is enough to prove

$$
\sup _{\xi \neq 0} \frac{\left|\widehat{f}_{0}(\xi)-\widehat{M}_{p}(\xi)\right|}{|\xi|^{\frac{2}{p+1}+\delta^{\prime}}}<+\infty
$$

and since $\widehat{f}_{0}$ and $\widehat{M}_{p}$ are even and bounded functions, it is enough to consider $0<\xi \leq 1$. The stationary state $M_{p}$ satisfies the same conditions (21) and (23) as $f_{0}$ with the same constants. Moreover, since

$$
\lim _{\xi \rightarrow 0^{+}} \frac{\partial_{\xi} \widehat{M}_{p}(\xi)}{\xi^{\frac{1-p}{p+1}}}=-\frac{2 \alpha}{p+1}
$$


and the same is true for $\widehat{f}_{0}$ as we proved in (28), we get

$$
\lim _{\xi \rightarrow 0^{+}} \frac{\partial_{\xi}\left(\widehat{f}_{0}-\widehat{M}_{p}\right)(\xi)}{\xi^{\frac{1-p}{p+1}}}=0 .
$$

We can therefore apply condition (23) to $\frac{\partial_{\xi}\left(\widehat{f}_{0}-\widehat{M}_{p}\right)(\xi)}{\xi^{\frac{1-p}{p+1}}}$ and pass to the limit for $\tau \rightarrow 0$ in order to get

$$
\left|\frac{\partial_{\xi}\left(\widehat{f}_{0}-\widehat{M}_{p}\right)(\xi)}{\xi^{\frac{1-p}{p+1}}}\right| \leq C|\xi|^{\delta}
$$

for a suitable $C>0$. Now, by Cauchy theorem and Hölder continuity (40) for $0<\xi \leq 1$ and $0<\delta^{\prime} \leq \delta$ we get

$$
\begin{aligned}
\frac{\left|\widehat{f}_{0}(\xi)-\widehat{M}_{p}(\xi)\right|}{\xi^{\frac{2}{p+1}+\delta^{\prime}}} & =\frac{1}{\frac{2}{p+1}+\delta^{\prime}} \frac{\left|\partial_{\xi}\left(\widehat{f}_{0}-\widehat{M}_{p}\right)(\tilde{\xi})\right|}{\tilde{\xi}^{\frac{1-p}{p+1}+\delta^{\prime}}} \\
& \leq \frac{C}{\frac{2}{p+1}+\delta^{\prime}} \frac{\tilde{\xi}^{\delta}}{\tilde{\xi}^{\delta^{\prime}}} \leq \bar{C}
\end{aligned}
$$

for $0<\tilde{\xi}<\xi$ and $\bar{C}>0$ and this ends the proof.

\section{Initial data with finite energy}

In the previous sections, we considered initial data $f_{0}$ with unbounded energy and we proved that, if these initial data belong to a suitable neighborhood of the stationary state, the corresponding solutions of the dissipative Kac equation (11)-(2) converge to the solution of a fractional Fokker-Planck equation when the collisions become grazing. In what follows, we briefly discuss the simpler case in which the initial data have bounded energy. As outlined in the Introduction, in this case any solution of (11)-(2) converges in large times to a Dirac delta function concentrated in $v=0$. This cooling behavior is maintained the grazing collision limit, where the collision operator reduces to a simple linear drift operator, while the diffusive term is lost. The limit equation is therefore

$$
\partial_{t} \widehat{f}(\xi, t)=-(p+1) \xi \partial_{\xi} \widehat{f}(\xi, t) .
$$

A simple calculation shows that if $\widehat{f}_{0}$ is the initial density, this equation has a unique explicit solution in the Fourier variable

$$
\widehat{f}(\xi, t)=\widehat{f_{0}}\left(\xi e^{-(p+1) t}\right),
$$

or, in the physical variable

$$
f(v, t)=e^{(p+1) t} f_{0}\left(e^{(p+1) t} v\right) .
$$

The next proposition deals with the aforementioned situation. 


\section{Proposition 8}

Assume $\left\{b_{\varepsilon}(\theta)\right\}_{\varepsilon>0} \subseteq L^{1}\left(\left[-\frac{\pi}{2}, \frac{\pi}{2}\right]\right)$ be a family of collision kernels satisfying Definition 1. Let $0<p \leq 1$ and let $f_{\varepsilon}(t) \in C^{1}\left([0,+\infty), L^{1}(\mathbb{R})\right)$ be the solutions of the dissipative Kac equations

$$
\partial_{t} \widehat{f}_{\varepsilon}(\xi, t)=2 \int_{0}^{\frac{\pi}{2}} b_{\varepsilon}(\theta)\left(\widehat{f}_{\varepsilon}\left(\xi \cos ^{p+1} \theta, t\right) \widehat{f}_{\varepsilon}\left(\xi \sin ^{p+1} \theta, t\right)-\widehat{f}_{\varepsilon}(\xi, t)\right) \mathrm{d} \theta
$$

where the even initial density $f_{0} \geq 0$ satisfies the normalization condition $\int f_{0}(v) \mathrm{d} v=1$ and has finite energy, $\int v^{2} f_{0}(v) \mathrm{d} v=1$.

Then,

$$
\lim _{\varepsilon \rightarrow 0} \sup _{t \geq 0, \xi \neq 0} \frac{\left|\widehat{f}_{\varepsilon}(\xi, t)-\widehat{f}(\xi, t)\right|}{|\xi|^{2}}=0
$$

where $f(t)$, given by (42) is the solution of the drift equation

$$
\partial_{t} \widehat{f}(\xi, t)=-(p+1) \xi \partial_{\xi} \widehat{f}(\xi, t)
$$

with the same initial data $f_{0}$.

Proof: As in the proof of Theorem 5 we consider only $0<\xi \leq R$. Since

$$
\begin{array}{r}
\partial_{t}\left(\widehat{f}_{\varepsilon}(\xi, t)-\widehat{f}(\xi, t)\right)=2 \int_{0}^{\frac{\pi}{2}} b_{\varepsilon}(\theta)\left(\widehat{f}_{\varepsilon}\left(\xi \cos ^{p+1} \theta, t\right) \widehat{f}_{\varepsilon}\left(\xi \sin ^{p+1} \theta, t\right)-\widehat{f}_{\varepsilon}(\xi, t)\right) \mathrm{d} \theta \\
+(p+1) \xi \partial_{\xi} \widehat{f}(\xi, t),
\end{array}
$$

we get

$$
\begin{aligned}
\partial_{t}\left(\widehat{f}_{\varepsilon}(\xi)-\right. & \widehat{f}(\xi))=-(p+1) \xi \partial_{\xi}\left(\widehat{f}_{\varepsilon}(\xi)-\widehat{f}(\xi)\right) \\
& +2 \int_{0}^{\frac{\pi}{2}} b_{\varepsilon}(\theta)\left(\widehat{f}_{\varepsilon}\left(\xi \cos ^{p+1} \theta\right) \widehat{f}_{\varepsilon}\left(\xi \sin ^{p+1} \theta\right)-\widehat{f}_{\varepsilon}(\xi)\right) \mathrm{d} \theta+(p+1) \xi \partial_{\xi} \widehat{f}_{\varepsilon}(\xi) .
\end{aligned}
$$

Therefore we have

$$
\partial_{t}\left(\frac{\widehat{f}_{\varepsilon}(\xi)-\widehat{f}(\xi)}{\xi^{2}}\right)=-(p+1) \frac{\partial_{\xi}\left(\widehat{f}_{\varepsilon}(\xi)-\widehat{f}(\xi)\right)}{\xi}+\frac{R_{\varepsilon}(\xi, t)}{\xi^{2}}
$$

where

$$
R_{\varepsilon}(\xi, t)=2 \int_{0}^{\frac{\pi}{2}} b_{\varepsilon}(\theta)\left(\widehat{f}_{\varepsilon}\left(\xi \cos ^{p+1} \theta\right) \widehat{f}_{\varepsilon}\left(\xi \sin ^{p+1} \theta\right)-\widehat{f}_{\varepsilon}(\xi)\right) \mathrm{d} \theta+(p+1) \xi \partial_{\xi} \widehat{f}_{\varepsilon}(\xi)
$$

Now, via a Taylor expansion with Lagrange reminder

$$
\begin{aligned}
\widehat{f}_{\varepsilon}\left(\xi \cos ^{p+1} \theta\right) \widehat{f}_{\varepsilon}\left(\xi \sin ^{p+1} \theta\right)-\widehat{f}_{\varepsilon}(\xi)= & \partial_{\xi} \widehat{f}_{\varepsilon}(\xi) \xi\left(\cos ^{p+1} \theta-1\right)+ \\
& \frac{\xi^{2}}{2}\left[\partial_{\xi}^{2} \widehat{f}_{\varepsilon}(\bar{\xi})\left(\cos ^{p+1} \theta-1\right)^{2}+\widehat{f}_{\varepsilon}(\xi) \partial_{\xi}^{2} \widehat{f}_{\varepsilon}(\tilde{\xi}) \sin ^{2(p+1)} \theta\right] \\
& +\frac{\xi^{3}}{2} \partial_{\xi} \widehat{f}_{\varepsilon}(\xi) \partial_{\xi}^{2} \widehat{f}_{\varepsilon}(\tilde{\xi})\left(\cos ^{p+1} \theta-1\right) \sin ^{2(p+1)} \theta \\
& +\frac{\xi^{4}}{4} \partial_{\xi}^{2} \widehat{f}_{\varepsilon}(\bar{\xi}) \partial_{\xi}^{2} \widehat{f}_{\varepsilon}(\tilde{\xi}) \sin ^{2(p+1)} \theta\left(\cos ^{p+1} \theta-1\right)^{2}
\end{aligned}
$$


where $\bar{\xi}$ and $\tilde{\xi}$ depend on $\xi, t, \varepsilon$ and $\theta$ and $\bar{\xi} \in\left(\xi, \xi \cos ^{p+1} \theta\right), \tilde{\xi} \in\left(0, \xi \sin ^{p+1} \theta\right)$. By assumption $(c)$ on $b_{\varepsilon}$,

$$
\begin{aligned}
R_{\varepsilon}(\xi, t) & =2 \xi \partial_{\xi} \widehat{f}_{\varepsilon}(\xi) \int_{0}^{\frac{\pi}{2}} b_{\varepsilon}(\theta)\left[\left(\cos ^{p+1} \theta-1\right)+\frac{p+1}{2} \sin ^{2} \theta\right] \mathrm{d} \theta \\
& +\xi^{2} \int_{0}^{\frac{\pi}{2}} b_{\varepsilon}(\theta)\left[\partial_{\xi}^{2} \widehat{f}_{\varepsilon}(\bar{\xi})\left(\cos ^{p+1} \theta-1\right)^{2}+\widehat{f}_{\varepsilon}(\xi) \partial_{\xi}^{2} \widehat{f}_{\varepsilon}(\tilde{\xi}) \sin ^{2(p+1)} \theta\right] \mathrm{d} \theta \\
& +\xi^{3} \partial_{\xi} \widehat{f}_{\varepsilon}(\xi) \int_{0}^{\frac{\pi}{2}} b_{\varepsilon}(\theta) \partial_{\xi}^{2} \widehat{f}_{\varepsilon}(\tilde{\xi})\left(\cos ^{p+1} \theta-1\right) \sin ^{2(p+1)} \theta \mathrm{d} \theta \\
& +\frac{\xi^{4}}{2} \int_{0}^{\frac{\pi}{2}} b_{\varepsilon}(\theta) \partial_{\xi}^{2} \widehat{f}_{\varepsilon}(\bar{\xi}) \partial_{\xi}^{2} \widehat{f}_{\varepsilon}(\tilde{\xi}) \sin ^{2(p+1)} \theta\left(\cos ^{p+1} \theta-1\right)^{2} \mathrm{~d} \theta
\end{aligned}
$$

Since the mass is conserved, and the energy decays, from (91) we obtain

$$
\int v^{2} f_{\varepsilon}(v, t) \mathrm{d} v=\exp \left\{-t \int_{-\pi / 2}^{\pi / 2} b_{\varepsilon}(\theta)\left(1-|\sin \theta|^{2+2 p}-|\cos \theta|^{2+2 p}\right) \mathrm{d} \theta\right\},
$$

Hence, for any $\xi \in \mathbb{R}, t \geq 0$ and $\varepsilon>0$

$$
\begin{aligned}
& \left|\widehat{f}_{\varepsilon}(\xi, t)\right| \leq\left|\widehat{f}_{\varepsilon}(0, t)\right|=\int f_{\varepsilon}(v, t) \mathrm{d} v=1, \\
& \left|\partial_{\xi}^{2} \widehat{f}_{\varepsilon}(\xi, t)\right| \leq \int v^{2} f_{\varepsilon}(v, t) \mathrm{d} v \leq 1, \\
& \left|\partial_{\xi} \widehat{f}_{\varepsilon}(\xi, t)\right| \leq \int|v| f_{\varepsilon}(v, t) \mathrm{d} v \leq\left(\int f_{\varepsilon}(v, t) \mathrm{d} v\right)\left(\int v^{2} f_{\varepsilon}(v, t) \mathrm{d} v\right)^{\frac{1}{2}} \leq 1 .
\end{aligned}
$$

Moreover, since $\partial_{\xi} \widehat{f}_{\varepsilon}(0, t)=0$ for all $t \geq 0$ we have

$$
\partial_{\xi} \widehat{f}_{\varepsilon}(\xi, t)=\partial_{\xi}^{2} \widehat{f}_{\varepsilon}\left(\xi_{*}, t\right) \xi
$$

for $\xi_{*}$ depending on $\xi, t$ and $\varepsilon$, which implies $\left|\frac{\partial_{\xi} \widehat{f}_{\varepsilon}(\xi, t)}{\xi}\right| \leq 1$ for all $\xi \neq 0, t \geq 0$ and $\varepsilon>0$. Thus, for $0<\xi \leq R, t \geq 0$ and $\varepsilon>0$ we get

$$
\left|\frac{R_{\varepsilon}(\xi, t)}{\xi^{2}}\right| \leq C(R) \int_{0}^{\frac{\pi}{2}} b_{\varepsilon}(\theta) g(\theta) \mathrm{d} \theta,
$$

where $C(R)>0$ is suitably chosen and

$$
\begin{aligned}
g(\theta)=\left|\left(\cos ^{p+1} \theta-1\right)+\frac{p+1}{2} \sin ^{2} \theta\right|+\left(\cos ^{p+1} \theta-1\right)^{2}+\sin ^{2(p+1)} \theta \\
\quad+\left|\cos ^{p+1} \theta-1\right| \sin ^{2(p+1)} \theta+\sin ^{2(p+1)} \theta\left(\cos ^{p+1} \theta-1\right)^{2} .
\end{aligned}
$$

Since $b_{\varepsilon}$ satisfies Definition 1 it follows

$$
\int_{0}^{\frac{\pi}{2}} b_{\varepsilon}(\theta) g(\theta) \mathrm{d} \theta=R_{\varepsilon} \rightarrow 0, \quad \varepsilon \rightarrow 0
$$


We can end the proof as in Theorem 5 and obtain

$$
\lim _{\varepsilon \rightarrow 0} \sup _{t \geq 0, \xi \neq 0} \frac{\left|\widehat{f}_{\varepsilon}(\xi, t)-\widehat{f}(\xi, t)\right|}{|\xi|^{2}}=0 .
$$

\section{Remark 9}

It is possible to adapt the previous proof to the classical conservative Kac equation considered in [Tos98]. In this case, we obtain a simpler proof of the convergence (in the previous Fourier based metric) of bounded energy solutions to the classical Fokker-Planck equation (10), when the collisions become grazing.

If we consider now the fractional Fokker-Planck equations (11), it is interesting to remark that the time behavior of the solutions corresponding to initial data with bounded energy is completely different. In fact, for any initial data $f_{0} \geq 0$ satisfying the normalization condition $\int f_{0}(v) \mathrm{d} v=1$ the solution of (11) converges to the corresponding stationary state $M_{p}=\exp \left\{-\alpha|\xi|^{2 /(1+p)}\right\}$ and that irrespective of how many finite moments the initial data possess. This implies in particular that the energy of the solution becomes immediately infinite, even if this energy was bounded at the beginning of the evolution.

\section{Proposition 10}

Let $0<p \leq 1, \alpha>0$ and let $f(t)$ be the solution of the Fokker-Planck equation

$$
\partial_{t} \widehat{f}(\xi, t)=-2 \alpha|\xi|^{\frac{2}{p+1}} \widehat{f}(\xi, t)-(p+1) \xi \partial_{\xi} \widehat{f}(\xi, t)
$$

with initial density $f_{0} \geq 0$, satisfying the normalization condition $\int f_{0}(v) \mathrm{d} v=1$. Then,

$$
\lim _{t \rightarrow+\infty}\left\|f(t)-M_{p}\right\|_{L^{1}}=0 .
$$

If moreover $f_{0}$ satisfies

$$
\int v f_{0}(v) \mathrm{d} v=0, \quad \int|v|^{\lambda} f_{0}(v) \mathrm{d} v<+\infty, \quad \lambda=\frac{2}{p+1},
$$

then

$$
\lim _{t \rightarrow+\infty} \sup _{\xi \neq 0} \frac{\left|\widehat{f}(\xi, t)-\widehat{M}_{p}(\xi)\right|}{|\xi|^{\frac{2}{p+1}}}=0 .
$$

Proof: Thanks to (20) we have to prove that

$$
\left\|\frac{1}{\beta(t)} f_{0}\left(\frac{\cdot}{\beta(t)}\right) * \frac{1}{\gamma(t)} M_{p}\left(\frac{\cdot}{\gamma(t)}\right)-M_{p}\right\|_{L^{1}} \longrightarrow 0, \quad t \rightarrow+\infty .
$$

To simplify notations, we will write

$$
\begin{aligned}
& f_{0, \beta}(v)=\frac{1}{\beta(t)} f_{0}\left(\frac{v}{\beta(t)}\right) \\
& M_{p, \gamma}(v)=\frac{1}{\gamma(t)} M_{p}\left(\frac{v}{\gamma(t)}\right) .
\end{aligned}
$$


Then

$$
\left\|f_{0, \beta} * M_{p, \gamma}-M_{p}\right\|_{L^{1}} \leq\left\|f_{0, \beta} * M_{p, \gamma}-f_{0, \beta} * M_{p}\right\|_{L^{1}}+\left\|f_{0, \beta} * M_{p}-M_{p}\right\|_{L^{1}} .
$$

Since $\beta(t) \rightarrow 0$ for $t \rightarrow+\infty$ and $f_{0} \in L^{1}$ with $\int f_{0}(v) \mathrm{d} v=1$, it is classical that $\left\|f_{0, \beta} * M_{p}-M_{p}\right\|_{L^{1}} \rightarrow 0$ [SW71, page 10]. Moreover, since

$$
\left\|f_{0, \beta} *\left(M_{p, \gamma}-M_{p}\right)\right\|_{L^{1}} \leq\left\|f_{0, \beta}\right\|_{L^{1}}\left\|M_{p, \gamma}-M_{p}\right\|_{L^{1}},
$$

and

$$
\int f_{0, \beta}(w) \mathrm{d} w=\int f_{0}(w) \mathrm{d} w=1,
$$

it is enough to prove that $a(t)=\left\|M_{p, \gamma}-M_{p}\right\|_{L^{1}}$ vanishes as time goes to infinity. For any given $R>0$, we have

$$
\begin{aligned}
a(t) & =\int\left|\frac{1}{\gamma(t)} M_{p}\left(\frac{v}{\gamma(t)}\right)-M_{p}(v)\right| \mathrm{d} v \\
& =\int_{|v| \leq R}\left|\frac{1}{\gamma(t)} M_{p}\left(\frac{v}{\gamma(t)}\right)-M_{p}(v)\right| \mathrm{d} v+\int_{|v|>R}\left|\frac{1}{\gamma(t)} M_{p}\left(\frac{v}{\gamma(t)}\right)-M_{p}(v)\right| \mathrm{d} v
\end{aligned}
$$

Let us consider first the second term. Since $\gamma(t)=\left(1-e^{-2 t}\right)^{\frac{p+1}{2}} \rightarrow 1$ for $t \rightarrow+\infty$, there is $t_{0}$ so that for $t \geq t_{0}$ we have $\frac{1}{2} \leq \gamma(t)<1$. So, for $t \geq t_{0}$

$$
\begin{aligned}
& \int_{|v|>R}\left|\frac{1}{\gamma(t)} M_{p}\left(\frac{v}{\gamma(t)}\right)-M_{p}(v)\right| \mathrm{d} v \\
& \leq \int_{|v|>R} \frac{1}{\gamma(t)} M_{p}\left(\frac{v}{\gamma(t)}\right) \mathrm{d} v+\int_{|v|>R} M_{p}(v) \mathrm{d} v \\
& =\int_{|v|>\frac{R}{\gamma(t)}} M_{p}(v) \mathrm{d} v+\int_{|v|>R} M_{p}(v) \mathrm{d} v \leq 2 \int_{|v|>R} M_{p}(v) \mathrm{d} v .
\end{aligned}
$$

Since $M_{p} \in L^{1}$, there is $R_{1}=R_{1}(\varepsilon)>0$ so that for $R \geq R_{1}$

$$
2 \int_{|v|>R} M_{p}(v) \mathrm{d} v<\frac{\varepsilon}{2}
$$

Let us come to the first integral in (46). For any $v \in \mathbb{R}$ we have

$$
\begin{aligned}
& \left|\frac{1}{\gamma(t)} M_{p}\left(\frac{v}{\gamma(t)}\right)-M_{p}(v)\right|=\left|\int\left(\widehat{M}_{p}(\gamma(t) \xi)-\widehat{M}_{p}(\xi)\right) e^{i v \xi} \mathrm{d} \xi\right| \\
& \leq \int\left|\widehat{M}_{p}(\gamma(t) \xi)-\widehat{M}_{p}(\xi)\right| \mathrm{d} \xi .
\end{aligned}
$$

Since $\lim _{t \rightarrow+\infty} \widehat{M}_{p}(\gamma(t) \xi)=\widehat{M}_{p}(\xi)$ for all $\xi$ and for $t \geq t_{0}$ we have $\frac{1}{2} \leq \gamma(t)<1$ and so $\widehat{M}_{p}(\gamma(t) \xi) \leq \widehat{M}_{p}(\xi / 2)$, by Lebesgue theorem there is $t_{1}=t_{1}(\varepsilon)>0$ so that for $t \geq \max \left(t_{0}, t_{1}\right)$ we have

$$
\int\left|\widehat{M}_{p}(\gamma(t) \xi)-\widehat{M}_{p}(\xi)\right| \mathrm{d} \xi \leq \frac{\varepsilon}{4 R}
$$


and so

$$
\int_{|v| \leq R}\left|\frac{1}{\gamma(t)} M_{p}\left(\frac{v}{\gamma(t)}\right)-M_{p}(v)\right| \mathrm{d} v \leq \frac{\varepsilon}{2} .
$$

Letting $\bar{t}=\max \left(t_{0}, t_{1}\right)$, the proof of (43) is completed.

To prove (45), we remark that conditions (44) imply

$$
\sup _{\xi \neq 0} \frac{\left|1-\widehat{f}_{0}(\xi)\right|}{|\xi|^{\frac{2}{p+1}}} \leq C
$$

for $C>0$ suitably chosen. Now, for $\xi \neq 0$ and $t \geq 0$

$$
\begin{aligned}
& \frac{\left|\widehat{f}(\xi, t)-\widehat{M}_{p}(\xi)\right|}{|\xi|^{\frac{2}{p+1}}}=\frac{\left|\widehat{f_{0}}\left(\xi e^{-(p+1) t}\right) e^{-\alpha|\xi|^{\frac{2}{p+1}}\left(1-e^{-2 t}\right)}-e^{-\alpha|\xi|^{\frac{2}{p+1}}}\right|}{|\xi|^{\frac{2}{p+1}}} \\
& \leq\left|\widehat{f}_{0}\left(\xi e^{-(p+1) t}\right)\right| \frac{\left|e^{-\alpha|\xi|^{\frac{2}{p+1}}\left(1-e^{-2 t}\right)}-e^{-\alpha|\xi|^{\frac{2}{p+1}}}\right|}{|\xi|^{\frac{2}{p+1}}}+e^{-\alpha|\xi|^{\frac{2}{p+1}}} \frac{\left|\widehat{f}_{0}\left(\xi e^{-(p+1) t}\right)-1\right|}{|\xi|^{\frac{2}{p+1}}} .
\end{aligned}
$$

Thanks to condition (47) on $f_{0}$ for $\xi \neq 0$ we get

$$
\begin{aligned}
\frac{\left|\widehat{f}(\xi, t)-\widehat{M}_{p}(\xi)\right|}{|\xi|^{\frac{2}{p+1}}} & \leq e^{-\alpha|\xi|^{\frac{2}{p+1}}\left(1-e^{-2 t}\right)} \frac{1-e^{-\alpha|\xi|^{\frac{2}{p+1}}} e^{-2 t}}{|\xi|^{\frac{2}{p+1}}}+e^{-2 t} \frac{1-\widehat{f}_{0}\left(\xi e^{-(p+1) t}\right)}{\left|\xi e^{-(p+1) t}\right|^{\frac{2}{p+1}}} \\
& \leq C(\alpha+1) e^{-2 t} \rightarrow 0, \quad t \rightarrow+\infty .
\end{aligned}
$$

Acknowledgment: The authors (A.P. and G.T) acknowledge support by MIUR project "Optimal mass transportation, geometrical and functional inequalities with applications". This paper has been written within the activities of the National Group of Mathematical Physics (GNFM) and of the National Group of Mathematical Analysis, Probability and Applications (GNAMPA) of INDAM.

\section{References}

[BWM00] D.A. Benson, S. W. Wheatcraft, M. M. Meerschaert. The fractional-order governing equation of Lévy motion, Water Resources Research, 36(6):1413$1423,2000$.

[Bob88] A. V. Bobylev. The theory of the nonlinear spatially uniform Boltzmann equation for Maxwell molecules. In Mathematical physics reviews, Vol. 7, volume 7 of Soviet Sci. Rev. Sect. C Math. Phys. Rev., pages 111-233. Harwood Academic Publ., Chur, 1988. 
[Cha98] A. S. Chaves. A fractional diffusion equation to describe Lévy flights. Phys. Lett. A, 239(1-2):13-16, 1998.

[CT98] J. A. Carrillo and G. Toscani. Exponential convergence toward equilibrium for homogeneous Fokker-Planck-type equations. Math. Methods Appl. Sci., 21(13):1269-1286, 1998.

[Des95] L. Desvillettes. About the regularizing properties of the non-cut-off Kac equation. Comm. Math. Phys., 168(2):417-440, 1995.

[Fel71] William Feller. An introduction to probability theory and its applications. Vol. II. Second edition. John Wiley \& Sons Inc., New York, 1971.

[Kac59] M. Kac. Probability and related topics in physical sciences, volume 1957 of With special lectures by G. E. Uhlenbeck, A. R. Hibbs, and B. van der Pol. Lectures in Applied Mathematics. Proceedings of the Summer Seminar, Boulder, Colo. Interscience Publishers, London-New York, 1959.

[KZB85] J. Klafter, G. Zumofen, A. Blumen. Concentration fluctuations in reaction kinetics, Journal of Chemical Physics, 82(7):3198-3206, 1985.

[LR79] R. G. Laha and V. K. Rohatgi. Probability theory. John Wiley \& Sons, New York-Chichester-Brisbane, 1979. Wiley Series in Probability and Mathematical Statistics.

[McK66] H. P. McKean, Jr. Speed of approach to equilibrium for Kac's caricature of a Maxwellian gas. Arch. Rational Mech. Anal., 21:343-367, 1966.

[MMM09] A. Mellet, S. Mischler, C. Mouhot. Fractional Diffusion Limit for Collisional Kinetic Equations. Arch. Rational Mech. Anal., 199:493-525, 2011.

[MFL02] F. J. Molz, III, G. J. Fix, III, and Silong Lu. A physical interpretation for the fractional derivative in Levy diffusion. Appl. Math. Lett., 15(7):907-911, 2002 .

[Mor54] D. Morgenstern. General existence and uniqueness proof for spatially homogeneous solutions of the Maxwell-Boltzmann equation in the case of Maxwellian molecules. Proc. Nat. Acad. Sci. U. S. A., 40:719-721, 1954.

[Mor55] D. Morgenstern. Analytical studies related to the Maxwell-Boltzmann equation. J. Rational Mech. Anal., 4:533-555, 1955.

[OBLU90] A. Ott, J. P. Bouchaud, D. Langevin, and W. Urbach. Anomalous diffusion in living polymers: A genuine Lévy flight? Phys. Rev. Lett. 65:2201-2204, 1990 .

[PT04] A. Pulvirenti and G. Toscani. Asymptotic properties of the inelastic Kac model. J. Statist. Phys., 114(5-6):1453-1480, 2004. 
[SBMW01] R. Schumer, D. A. Benson, M.M. Meerschaert, S.W. Wheatcraft. Eulerian derivation of the fractional advection-dispersion equation, Journal of Contaminant Hydrology 48:69-88, 2001.

[SW71] E. M. Stein and G. Weiss. Introduction to Fourier analysis on Euclidean spaces. Princeton University Press, Princeton, N.J., 1971. Princeton Mathematical Series, No. 32.

[SWK87] M. F. Shlesinger, B. J. West, and J. Klafter. Lévy dynamics of enhanced diffusion: application to turbulence. Phys. Rev. Lett., 58(11):1100-1103, 1987.

[SZK93] M. F. Shlesinger, G. M. Zaslavsky, J. Klafter, Strange kinetics. Nature, 363:31-37, 1993.

[Tos98] G. Toscani. The grazing collisions asymptotics of the non-cut-off Kac equation. RAIRO Modél. Math. Anal. Numér., 32(6):763-772, 1998.

[TV99] G. Toscani and C. Villani. Sharp entropy dissipation bounds and explicit rate of trend to equilibrium for the spatially homogeneous Boltzmann equation. Comm. Math. Phys., 203(3):667-706, 1999.

[Vil98] C. Villani. On a new class of weak solutions to the spatially homogeneous Boltzmann and Landau equations. Arch. Rational Mech. Anal., 143(3):273307, 1998.

[Vil06] C. Villani. Mathematics of granular materials. J. Stat. Phys., 124(2-4):781$822,2006$.

[Wil51] E. Wild. On Boltzmann's equation in the kinetic theory of gases. Proc. Cambridge Philos. Soc., 47:602-609, 1951. 